\title{
Integrated analysis highlights multiple long non-coding RNAs and their potential roles in the progression of human esophageal squamous cell carcinoma
}

\author{
CHENG-YUN LI ${ }^{1 *}$, WEN-WEN ZHANG $^{1 *}$, JI-LIAN XIANG $^{2}$, XING-HUA WANG ${ }^{3}$, \\ JUN-LING WANG ${ }^{1}$ and JIN LI ${ }^{1}$ \\ ${ }^{1}$ Department of Toxicology, School of Public Health, Lanzhou University; \\ ${ }^{2}$ Department of Gastroenterology, Third People's Hospital of Gansu Province, Lanzhou, Gansu 730000; \\ ${ }^{3}$ Department of Gastrointestinal Surgery, Gansu Wuwei Tumor Hospital, Wuwei, Gansu 733000, P.R. China
}

Received April 22, 2019; Accepted September 20, 2019

DOI: $10.3892 /$ or.2019.7377

\begin{abstract}
Esophageal squamous cell carcinoma (ESCC) is a prevalent aggressive malignant tumor with poor prognosis. Investigations into the molecular changes that occur as a result of the disease, as well as identification of novel biomarkers for its diagnosis and prognosis, are urgently required. Long non-coding RNAs (lncRNAs) have been reported to play a critical role in tumor progression. The present study performed data mining analyses for ESCC via an integrated study of accumulated datasets and identification of the differentially expressed lncRNAs from the Gene Expression Omnibus (GEO) and The Cancer Genome Atlas (TCGA) databases. The identified intersection of differentially expressed genes (lncRNAs, miRNAs and mRNAs) in ESCC tissues between the GEO and TCGA datasets was investigated. Based on these intersected lncRNAs, the present study constructed a competitive endogenous RNA (ceRNA) network of lncRNAs in ESCC. A total of 81 intersection lncRNAs were identified; 67 of these were included in the ceRNA network. Functional analyses revealed that these 67 key lncRNAs primarily dominated cellular biological processes. The present study then analyzed the associations between the expression levels of these 67 key lncRNAs and the clinicopathological characteristics of the ESCC patients, as well as their survival time using TCGA. The results revealed that 31 of these IncRNAs were associated with tumor grade, tumor-node-metastasis (TNM) stage and lymphatic metastasis status $(\mathrm{P}<0.05)$. In addition, 15 key lncRNAs were
\end{abstract}

Correspondence to: Dr Cheng-Yun Li, Department of Toxicology, School of Public Health, Lanzhou University, 199 Donggang West Road, Lanzhou, Gansu 730000, P.R. China

E-mail: 13919995713@163.com

${ }^{*}$ Contributed equally

Key words: esophageal cancer, long non-coding RNA, clinical characteristics, overall survival demonstrated to be associated with survival time $(\mathrm{P}<0.05)$. Finally, 5 key lncRNAs were selected for validation of their expression levels in 30 patients newly diagnosed with ESCC via reverse transcription-quantitative PCR (RT-qPCR). The results suggested that the fold changes in the trends of up- and downregulation between GEO, TCGA and RT-qPCR were consistent. In addition, it was also demonstrated that a select few of these 5 key lncRNAs were significantly associated with TNM stage and lymph node metastasis $(\mathrm{P}<0.05)$. The results of the clinically relevant analysis and the aforementioned bioinformatics were similar, hence proving that the bioinformatics analysis used in the present study is credible. Overall, the results from the present study may provide further insight into the functional characteristics of lncRNAs in ESCC through bioinformatics integrative analysis of the GEO and TCGA datasets, and reveal potential diagnostic and prognostic biomarkers for ESCC.

\section{Introduction}

According to statistics from the World Health Organization (WHO) in 2015, esophageal cancer ranks seventh in terms of global cancer incidence $(572,000$ new cases per year) and sixth overall in global cancer-associated mortality $(509,000$ deaths) (1). Approximately $85 \%$ of all esophageal cancer cases are esophageal squamous cell carcinoma (ESCC), and the most common cause of death is metastasis (2). Tumor staging and grading systems are important in the clinical diagnosis of cancer, but are currently not adequate for prognosis and prediction of the disease (3). Frequently, when patients with ESCC are diagnosed in clinical practice, the majority have already progressed to advanced stage and/or lymphatic metastasis. Furthermore, the survival rate in patients with ESCC is very poor, and there is a lack of specific biomarkers for early diagnosis and prognosis (4). Therefore, more efficient and accurate ESCC diagnostic and prognostic biomarkers are urgently required in order to improve these areas, including screening for the early stages of ESCC, as well as new effective treatment methods.

Accumulating evidence has revealed that dysregulated long non-coding RNAs (lncRNAs) play a number 
of key biological roles in the progression of various types of cancer $(5,6)$. An increasing number of studies have suggested that aberrant expression of lncRNAs in ESCC is closely associated with histological type, tumor-node-metastasis (TNM) stage, lymph node metastasis and prognosis $(7,8)$.

ESCC is a multistep disease, which involves multiple interactions between genetic and environmental factors. Furthermore, lncRNAs play an important regulatory role in epigenetics. Investigating the ESCC-associated alterations of lncRNAs may aid the identification of valuable biomarkers for ESCC diagnosis and prognosis. Previous studies have primarily focused on the diagnostic and prognostic performance of a small portion of lncRNAs in ESCC, but a larger number of lncRNAs remain unexplored. Therefore, elucidating the functions of dysregulated lncRNAs, particularly in ESCC, is currently an important research topic.

Currently, high-throughput RNA sequencing technologies are being widely used for the detection of lncRNA alterations in carcinogenesis and screening for potential biomarkers of numerous diseases (9). However, the small sample sizes used for microarray detection often present a bias toward the identification of ESCC-associated lncRNAs due to lack of RNA sequencing data and, therefore, often generate errors (10). By using large sample sizes that integrate multiple RNA sequencing datasets, sufficient information regarding patients with ESCC can be obtained, thereby providing more convincing results. Thus far, with the advent of high-throughput RNA sequencing technologies, The Cancer Genome Atlas (TCGA; https://portal. gdc.cancer.gov/) and Gene Expression Omnibus (GEO; https://www.ncbi.nlm.nih.gov/geo/) database platforms have allowed the collected RNA sequencing data from microarray chips to be uploaded and standardized for quality control (11). Therefore, identification of the ESCC-associated lncRNAs would be more reliable with the use of large sample sizes integrating multiple analyses from different RNA sequencing databases.

The RNA sequencing databases obtained from the tissues of patients with ESCC are expected to provide a novel analysis strategy to identify diagnostic and prognostic biomarkers for this disease. In the present study, data mining analyses for ESCC were performed by integrating the significant differences in RNA obtained from the GEO and TCGA databases. Through these efforts, co-differentially expressed lncRNAs in ESCC may be identified. Based on these IncRNAs, subsequent analyses were performed, including gene functional enrichment analyses, competing endogenous RNA network construction, assessment of the association between differentially expressed lncRNAs and the clinicopathological characteristics of patients with ESCC, and survival analyses. Finally, reverse transcription-quantitative PCR (RT-qPCR) was used to validate the bioinformatics analysis results in the tumor and adjacent non-tumor tissues of 30 patients with newly diagnosed ESCC. This new approach may contribute to an improved method for identifying potential lncRNA biomarkers for the diagnosis of ESCC, as well as its classification and prediction of prognosis.

\section{Materials and methods}

Microarray dataset collection. The present study collected RNA sequencing expression datasets (lncRNAs, miRNAs and mRNAs) comprising tissue samples from 802 patients with ESCC, which included cancer tissues and adjacent normal esophageal tissues from the GEO genomics database. The RNA sequencing datasets of patients with ESCC were downloaded from the GSE23400 (12) (208 tissue samples: https://www.ncbi.nlm. nih.gov/geo/query/acc.cgi?acc=GSE23400), GSE26886 (13) (69 tissue samples: https://www.ncbi.nlm.nih. gov/geo/query/acc.cgi?acc=GSE26886), GSE45670 (14) (38 tissue samples: https://www.ncbi.nlm.nih. gov/geo/query/acc.cgi?acc=GSE45670), GSE97049 (14 tissue samples: https://www.ncbi.nlm.nih. gov/geo/query/acc.cgi?acc=GSE97049), GSE6188 (15) (257 tissue samples: https://www.ncbi.nlm.nih. gov/geo/query/acc.cgi?acc=GSE6188) andGSE55856(16) (216 tissue samples: https://www.ncbi.nlm.nih.gov/geo/query/acc. cgi?acc=GSE55856) datasets. All the aforementioned GEO datasets were obtained from Affymetrix Human Genome Array platforms. A total of 312 patients with ESCC were obtained from the TCGA database (up to November 1, 2018). Annotation information of the RNA sequencing datasets was obtained using the Affymetrix Human Genome Array platforms. The present study was fully compliant with the publication guidelines provided by the GEO and TCGA databases. The details of GEO and TCGA database ESCC patient tissue RNA sequencing datasets, sample descriptions and clinicopathological characteristics are provided in Tables I and II. A flow diagram of the integrated bioinformatics analysis from the GEO and TCGA databases is provided in Fig. 1.

Annotation of microarray probes. In order to enhance the comparability of the GEO database RNA sequencing data, the signature values of the GSE23400, GSE26886, GSE45670, GSE97049, GSE6188 and GSE55856 RNA sequencing datasets were downloaded and converted to new comparable transcripts. Probe reannotation methods were used to obtain a standard measurement of the RNA sequencing expression profiles. Once the aforementioned ESCC RNA sequencing probe signature values and probe IDs were downloaded from the GEO database, they underwent a mapped detection analysis in a database of human genomes, and those that were mismatched were excluded. The remaining probe positions on the chromosomes were identified using the GENCODE tool (https://www.gencodegenes.org/). Probes matched to both protein-coding genes and lncRNAs were also excluded. If multiple probes matched the same gene, the median values were instead used as the expression level of that gene. In addition, prior to any further analyses, RNA sequencing data normalization was performed to ensure the data were fully compliant with the publication guidelines of TCGA.

Integration of microarray data and differential expression analysis. All the ESCC tissue samples from the GEO database were downloaded from three or more datasets in order to enlarge the sample number and avoid generating less 
Table I. Details of ESCC studies and RNA sequencing microarray datasets from the GEO database.

\begin{tabular}{|c|c|c|c|}
\hline GSE & Publication & RNA sequencing styles & Sample size for each group \\
\hline \multirow[t]{2}{*}{ GSE23400 } & Clinical cancer research & $\operatorname{lncRNA}$ & Tumor, 104 \\
\hline & & mRNA & Adjacent normal tissues, 104 \\
\hline \multirow[t]{2}{*}{ GSE26886 } & BMC Cancer & lncRNA & Tumor, 35 \\
\hline & & mRNA & Adjacent normal tissues, 34 \\
\hline \multirow[t]{2}{*}{ GSE45670 } & Annals of oncology & lncRNA & Tumor, 28 \\
\hline & & mRNA & Adjacent normal tissues, 10 \\
\hline \multirow[t]{2}{*}{ GSE97049 } & Unrecorded & miRNA & Tumor, 7 \\
\hline & & & Adjacent normal tissues, 7 \\
\hline \multirow[t]{2}{*}{ GSE6188 } & Cancer research & miRNA & Tumor, 153 \\
\hline & & & Adjacent normal tissues, 104 \\
\hline \multirow[t]{2}{*}{ GSE55856 } & Gut & miRNA & Tumor, 108 \\
\hline & & & Adjacent normal tissues, 108 \\
\hline
\end{tabular}

ESCC, esophageal squamous cell carcinoma; GEO, Gene Expression Omnibus.

Table II. Clinical information and samples size for TCGA ESCC datasets.

\begin{tabular}{|c|c|c|c|}
\hline Variables & $\begin{array}{l}\text { Total cases, } \\
\mathrm{n}=312(\%)\end{array}$ & $\begin{array}{c}\text { Alive, } \\
\mathrm{n}=138(\%)\end{array}$ & $\begin{array}{l}\text { Deceased, } \\
\mathrm{n}=174(\%)\end{array}$ \\
\hline \multicolumn{4}{|l|}{ Sex } \\
\hline Male & $211(67.63)$ & $83(60.14)$ & $128(73.56)$ \\
\hline Female & $101(32.37)$ & $55(39.86)$ & $46(26.44)$ \\
\hline \multicolumn{4}{|l|}{ Race } \\
\hline White & $162(51.92)$ & $5(39.86)$ & 107 (61.49) \\
\hline Asia & $127(40.71)$ & $68(49.28)$ & $59(33.91)$ \\
\hline Black & $23(7.37)$ & 15 (10.87) & $8(4.60)$ \\
\hline \multicolumn{4}{|l|}{ Age, years } \\
\hline$\leq 50$ & $86(27.56)$ & $53(38.41)$ & 33 (18.97) \\
\hline$>50$ & $226(72.44)$ & 85 (61.59) & $141(81.03)$ \\
\hline \multicolumn{4}{|l|}{ Tumor grade } \\
\hline GI & $49(15.71)$ & $31(22.46)$ & $18(10.34)$ \\
\hline GII & $146(46.79)$ & $48(34.78)$ & $98(56.32)$ \\
\hline GIII-IV & $117(37.50)$ & $59(42.75)$ & $58(33.33)$ \\
\hline \multicolumn{4}{|l|}{ TNM stage } \\
\hline $\mathrm{I} / \mathrm{II}$ & $99(31.73)$ & $30(21.74)$ & 69 (39.66) \\
\hline III/IV & $213(68.27)$ & 108 (78.26) & $105(60.34)$ \\
\hline \multicolumn{4}{|l|}{ Lymph } \\
\hline \multicolumn{4}{|l|}{ node status } \\
\hline No metastasis & $35(11.22)$ & $32(23.19)$ & $3(1.72)$ \\
\hline Metastasis & 277 (88.78) & $106(76.81)$ & $171(98.28)$ \\
\hline
\end{tabular}

TCGA, The Cancer Genome Atlas; ESCC, esophageal squamous cell carcinoma.

reliable results. A differential analysis was then separately performed for each dataset, comparing ESCC tumor tissues to adjacent normal tissues using the limma R 3.4.4 software (https://www.r-project.org/) [false discovery rate (FDR) $<0.05$, fold change $>2, \mathrm{P}<0.05]$. Subsequently, overlapping subclass analyses were used to identify the separate co-differentially expressed genes in each dataset, including lncRNAs, miRNAs and mRNAs, with the Venn 2.1 tool (http://bioinfogp.cnb. csic.es/tools/venny/index.html). The integrated ESCC tissue dysregulated lncRNA, miRNA and mRNA lists were saved for further analysis.

TCGA database provided the normalized RNA sequencing data, which included lncRNAs and mRNAs from patients with ESCC using the RNASeqV2 system. Furthermore, ESCC level 3 normalized miRNA sequencing data (Illumina HiSeq 2000 microRNA sequencing platforms) were also downloaded from TCGA. The significantly differentially expressed lncRNAs, mRNAs and miRNAs in the 312 tumor tissues and 47 adjacent non-tumor esophageal epithelial tissues in patients with ESCC were then analyzed (FDR $<0.05$, fold change $>2, \mathrm{P}<0.05$ ). The significantly different lncRNAs, mRNAs and miRNAs were selected for further analysis.

Finally, according to the fold changes of differentially expressed lncRNAs, mRNAs and miRNAs in ESCC tissues from the GEO and TCGA databases, the common genes were selected for subsequent analysis.

Construction of the competing endogenous (ce) RNA network. In the present study, an IncRNA, miRNA and mRNA ceRNA network was built based on the theory that lncRNAs can regulate miRNA abundance by sequestration binding, acting as 'miRNA sponges' through miRNA binding to the mRNAs and negatively regulating gene expression. Common ESCC tissues with significantly differentially expressed lncRNAs, mRNAs and miRNAs (FDR $<0.05$, fold change $>2, \mathrm{P}<0.05$ ) were selected to build the ceRNA network, in which the fold changes of genes were rooted in the TCGA database, in order to investigate whether these intersection IncRNAs, mRNAs and miRNAs were involved in ceRNA regulation. MiRcode (https://omictools.com/mircode-tool), miRanda (http://www.microrna.org/microrna/home.do) and 


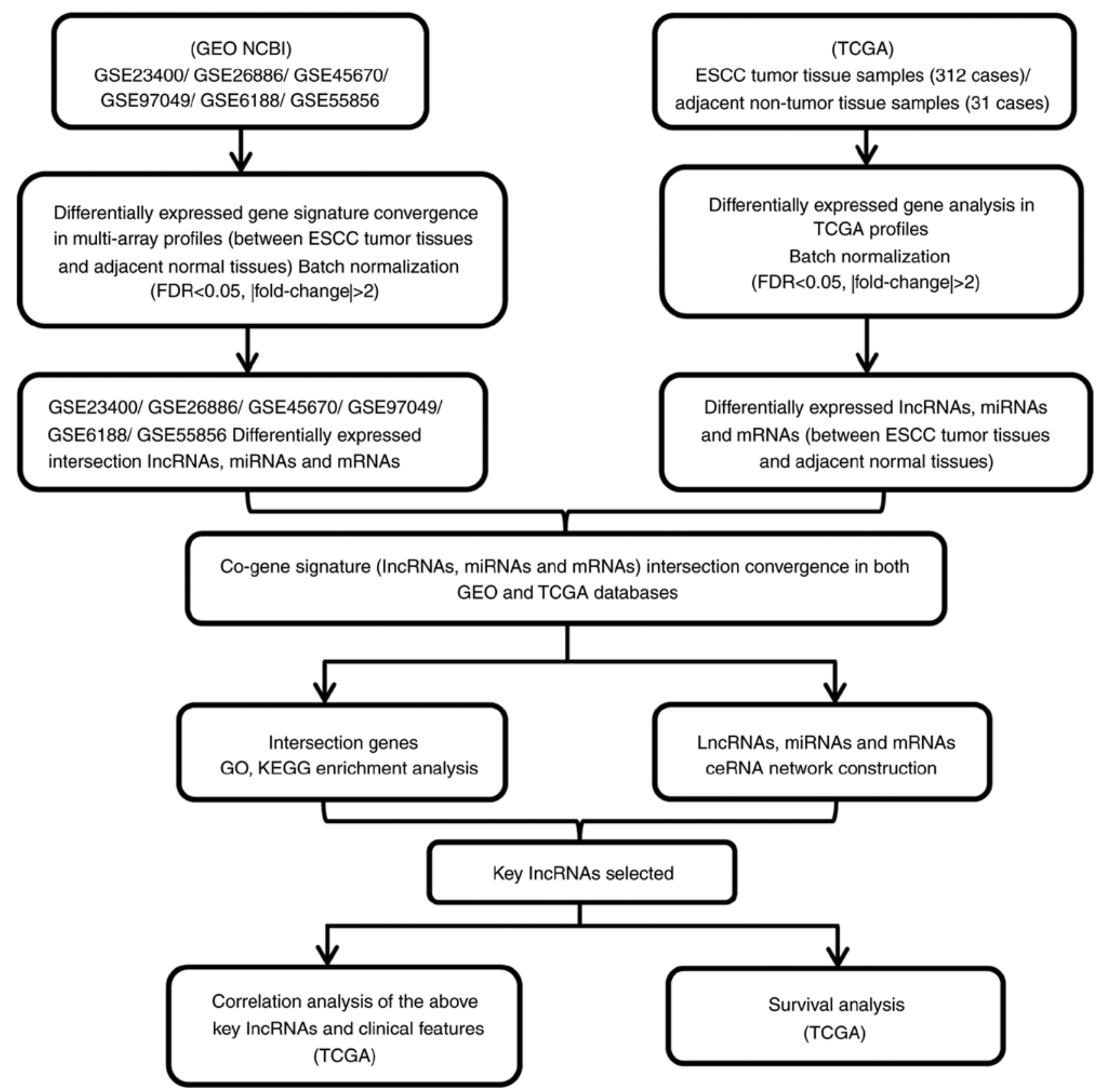

Figure 1. Flowchart for integrated bioinformatics analysis of ESCC publicly available RNA sequencing datasets from the GEO and TCGA databases. ESCC, esophageal squamous cell carcinoma; GEO, Gene Expression Omnibus; TCGA, The Cancer Genome Atlas.

Targetscan (http://www.targetscan.org/) were used to predict the miRNA target lncRNAs and miRNA-mRNA interactions in the different databases. Finally, the predicted miRNA target genes and the significantly differentially expressed intersection genes in the GEO and TCGA databases were used to build the ceRNA network. A flow chart for the lncRNA, miRNA and mRNA ceRNA network construction is presented in Fig. 2.

During this process, the lncRNA, miRNA and mRNA ceRNA network was constructed using the fold change data of the significantly expressed RNA of patients with ESCC from TCGA database using the R package. Pearson's correlation matrix models were used to identify the potential relevance of all pair-wise genes. Finally, based on the weighted adjacency matrix, the network connectivity of genes with other genes was investigated, and the ceRNA network was built using Cytoscape software (version 3.0.).

Gene Ontology (GO) and Kyoto Encyclopedia of Genes and Genomes (KEGG) pathway analysis. In order to investigate the potential gene functional enrichment and signaling pathway regulation of the consensus mRNAs that were involved in the ceRNA network, GO (http://www.geneontology.org) and KEGG pathway analyses were performed. The mRNAs that were involved in the ceRNA network were uploaded to the GO database to investigate the enriched molecular functions of these mRNAs. Upregulated and downregulated mRNAs from the ceRNA network were analyzed. Furthermore, the KEGG tool (http://www. kegg.jp/) was used to identify the potential regulated signaling pathways 


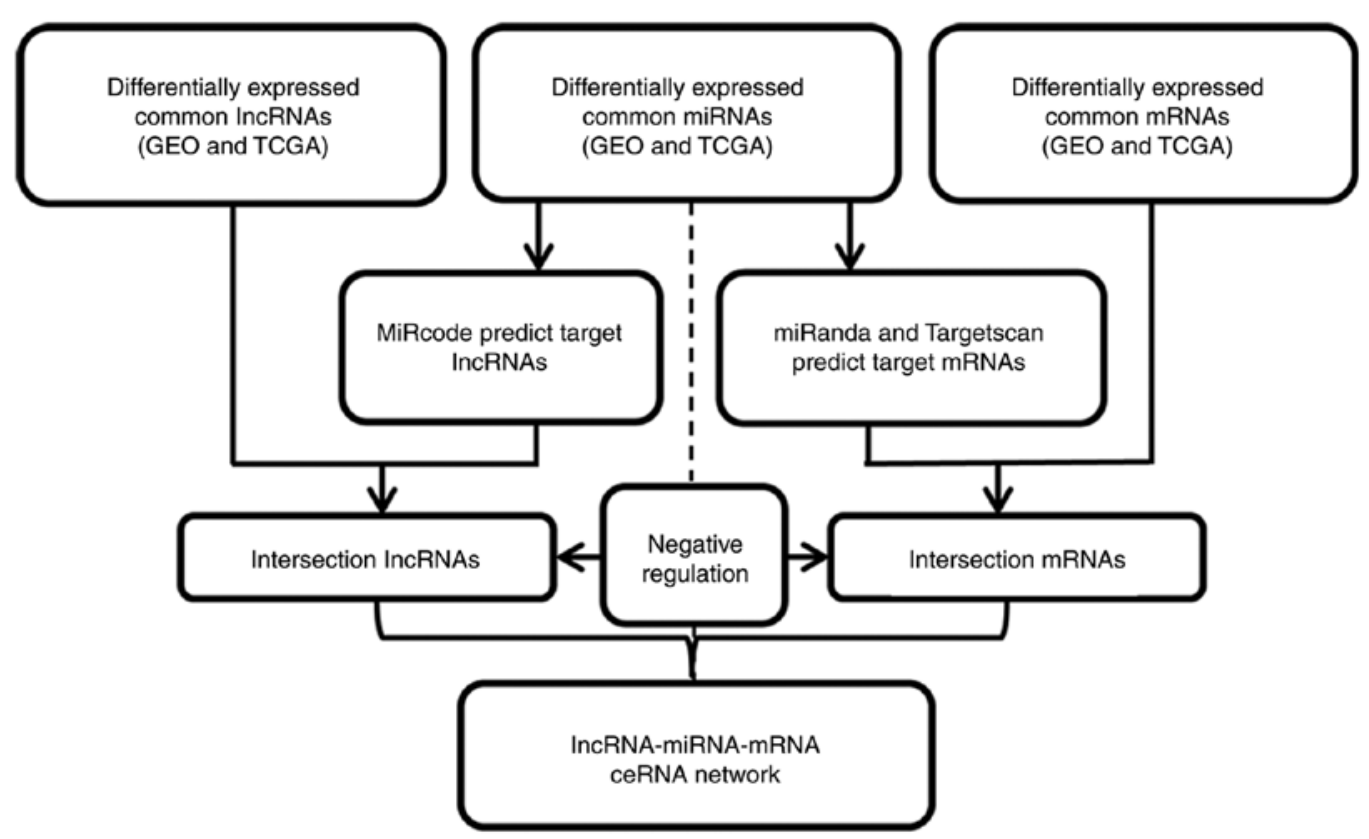

Figure 2. Flowchart for lncRNA, miRNA and mRNA ceRNA network construction. IncRNA, long non-coding RNA.

of these genes. R software was used to visualize the GO and KEGG results.

Association between ceRNA network key lncRNAs and ESCC clinical status from TCGA. Based on the co-expression of the IncRNAs, miRNAs and mRNAs in the ceRNA network, the key lncRNAs that were involved in the network were selected as target lncRNAs potentially associated with ESCC progression. Subsequently, the potential association between ceRNA network key lncRNAs and TCGA ESCC patients' clinicopathological characteristics were investigated, which included sex, TNM stage, tumor grade, lymphatic metastasis status and pathological stage using multiple linear regression analysis.

Kaplan-Meier survival analysis. In order to investigate whether the key lncRNAs that were involved in the ceRNA network were associated with the TCGA database status, a Kaplan-Meier survival analysis of the ESCC patients from TCGA was performed. Based on the TCGA ESCC patient datasets, the fold changes of the aforementioned selected key lncRNAs in the cancer tissues and overall survival rates of patients with ESCC were determined using the GEPIA tool (http:/gepia.cancer-pku.cn/). Kaplan-Meier survival analysis parameters were calculated using the publicly available TCGA ESCC patient datasets and GEPIA tools. The survival distributions of different TCGA with ESCC, and the expression changes in key lncRNAs, were examined using Kaplan-Meier analysis, log-rank test and hazard ratio (HR).

Preparation of human ESCC samples and RT-qPCR validation of bioinformatics analysis results. Next, 30 tumor and paired non-tumor esophageal tissues from patients with ESCC were collected from the Gansu Wuwei Tumor Hospital (Wuwei, China); the patients were aged 40-75 years. The samples were collected and stored at $-80^{\circ} \mathrm{C}$. All patients were diagnosed with ESCC according to their pathological examination results.
Clinical basic information and informed consent forms were obtained from all ESCC patients (Table III). The collection of the tumor samples from patients with ESCC was approved by the Ethics Committee of the Gansu Wuwei Tumor Hospital.

Total RNA was isolated from tissue samples using TRIzol ${ }^{\circledR}$ reagent (Invitrogen; Thermo Fisher Scientific, Inc.). Reverse Transcription Kit (Promega Corporation) and GoTaq ${ }^{\circledR}$ qPCR Master Mix of Power SYBR ${ }^{\circledR}$ Green (Promega Corporation) were used to synthesize cDNA and perform RT-qPCR analysis. The reaction was performed at $95^{\circ} \mathrm{C}$ for $2 \mathrm{~min}$, followed by 40 cycles at $95^{\circ} \mathrm{C}$ for $15 \mathrm{sec}, 60^{\circ} \mathrm{C}$ for $30 \mathrm{sec}$ and $72^{\circ} \mathrm{C}$ for $30 \mathrm{sec}$. A dissociation curve was analyzed from $60-95^{\circ} \mathrm{C}$. Finally, RT-qPCR was performed using the Step One Plus ${ }^{\mathrm{TM}}$ PCR System (Applied Biosystems; Thermo Fisher Scientific, Inc.). RT-qPCR relative fold change results were calculated using the $2^{-\Delta \Delta \mathrm{Cq}}$ method (17).

Statistical analysis. All ESCC tissue RNA sequencing datasets from the GEO database were obtained from at least three independent datasets. $\mathrm{R}$ software was used to normalize the RNA sequencing data and compare significantly differentially expressed genes. Cytoscape 3.0 and GEPIA software tools were used to construct the ceRNA network and perform the survival analysis. Two-tailed Student's t-test was used to assess the differences between subgroups. $\mathrm{P}<0.05$ was considered to indicate a statistically significant difference.

\section{Results}

Convergence of gene expression signatures across the different datasets of patients with ESCC from GEO. The ESCC RNA sequencing data and other information were obtained from the GEO database. In order to increase the veracity and reliability of the signal values, and also decrease the possibility of false-positives, three independent datasets were downloaded, including ESCC tissue lncRNA, miRNA and mRNA 
Table III. Demographic and clinical characteristics of 30 patients with ESCC.

\begin{tabular}{lc}
\hline Variables & Total cases, $\mathrm{n}=30(\%)$ \\
\hline $\begin{array}{l}\text { Age, years } \\
\text { (mean } \pm \text { standard deviation) }\end{array}$ & $59 \pm 9.72$ \\
Sex & \\
Male & $20(66.67)$ \\
Female & $10(33.33)$ \\
TNM stage & \\
I/II & $8(26.67)$ \\
III/IV & $22(73.33)$ \\
Lymph node status & \\
No metastasis & $9(30.00)$ \\
Metastasis & $21(70.00)$ \\
\hline
\end{tabular}

ESCC, esophageal squamous cell carcinoma.

sequencing results. Differentially expressed genes were identified using the limma package according to the threshold of $\mathrm{FDR}<0.05$, fold change $>2$ and $\mathrm{P}<0.05$. Volcano plots and Venn analysis revealed the number of differentially expressed genes identified from each dataset (Fig. 3). The GSE23400 dataset contained 5,310 differentially expressed genes, including 792 differentially expressed lncRNAs and 4,518 mRNAs. The GSE26886 dataset contained 8,747 differentially expressed genes, including 1,340 differentially expressed lncRNAs and 7,407 mRNAs. The GSE45670 dataset contained 6,336 differentially expressed genes, including 914 differentially expressed lncRNAs and 5,422 mRNAs. The GSE97049 dataset contained 178 differentially expressed miRNAs. The GSE6188 dataset contained 198 differentially expressed miRNAs. Finally, the GSE55856 dataset contained 198 differentially expressed miRNAs. The detailed information of these six datasets and the number of differentially expressed genes identified from each dataset are presented in Table IV.

Following the Venn tool intersection, differentially expressed genes were analyzed, and it was demonstrated that there were 108 common lncRNAs, 1,234 mRNAs and 45 miRNAs with significantly changed levels in ESCC tissues from the GEO database (Fig. 3; Table IV). Among those, 59 lncRNAs, 779 mRNAs and 30 miRNAs were upregulated, whereas 49 lncRNAs, 455 mRNAs and 15 miRNAs were downregulated. Detailed information on these common significantly differentially expressed genes in ESCC tissues from GEO database can be found in Tables SI-SII.

ESCC tissues with co-differentially expressed genes identified in the GEO and TCGA databases. The ESCC patient tissues RNA sequencing results and their clinicopathological information were collected from TCGA database. Compared with the tumor and non-tumor tissue RNA sequencing results of patients with ESCC from TCGA, we identified a total of 889 differentially expressed lncRNAs (597 upregulated and 292 downregulated, 4,796 differentially expressed
mRNAs (3,170 upregulated and 1,626 downregulated), and 438 differentially expressed mRNAs (299 upregulated and 139 downregulated) (Table IV). The Venn diagram in Fig. 4 presents the intersections of genes between the GEO and TCGA databases. A total of 81 co-differentially expressed lncRNAs, 39 miRNAs and 357 mRNAs were identified (Fig. 4 and Table V).

Construction of ceRNA network. In order to determine whether the intersection of co-differentially expressed lncRNAs, miRNAs and mRNAs in GEO and TCGA (Table V) exist in competing endogenous regulating relationships, the intersected 81 lncRNAs, 39 miRNAs and 357 mRNAs were applied to construct the ceRNA network. The aforementioned 39 miRNAs target lncRNAs and mRNAs were then predicted based on MiRcode, miRanda and Targetscan. Subsequently, the intersection genes of the abovementioned miRNAs target predicted the lncRNAs and mRNAs to build the ceRNA network (Table V). The ceRNA network was visualized using Cytoscape software (version 3.0). The results revealed that there were 67 lncRNAs, 37 miRNAs and 80 mRNAs involved in the ceRNA network (Fig. 5). The detailed information of ceRNA network is presented in Table SIII.

Functional analysis of $m R N A s$ in the ceRNA network. The present study identified $67 \mathrm{lncRNAs}, 37 \mathrm{miRNAs}$ and $80 \mathrm{mRNAs}$ involved in the ceRNA network. Therefore, it may be suggested that the co-differentially expressed genes in the ESCC tissues that were included in the ceRNA network may play key roles in ESCC progression. Based on these suggestions, the present study investigated the potential biological regulatory functions of these 80 mRNAs that were involved in the ceRNA network via GO enrichment of functions and KEGG pathway analyses. The upregulated and downregulated intersected mRNAs were further analyzed. The results suggested that the most enriched GO function by upregulated mRNAs was 'Regulation of biological process (GO:0050789)'. The most enriched GO function by downregulated mRNAs was 'Single-organism cellular process (GO:0044763)' (Fig. 6). The KEGG pathway analysis indicated 25 signaling pathways involved in regulating upregulated mRNAs, and the most enriched pathway was 'MicroRNAs in cancer (hsa05206)'. In addition, 95 signaling pathways were involved in regulating downregulated mRNAs, and the most enriched pathway was 'Insulin secretion (hsa04911)'. Some of these signaling pathways, such as the 'Wnt signaling pathway', were involved in the progression of ESCC (18), the 'MAPK signaling pathway' was revealed as a key pathway affect ing esophageal carcinoma cell proliferation and apoptosis (19), and the 'mTOR signaling pathway' was involved in the progression of ESCC (20). Furthermore, 'Bladder cancer, Non-small-cell lung cancer, Pathways in cancer and PI3K-AKT signaling pathway' were also reported as cancer-associated signaling pathways (21-24) (Fig. 7).

Association between lncRNA signature and the clinical characteristics of patients with ESCC. The aforementioned 67 lncRNAs that were involved in the ceRNA network were selected and further investigated in order to identify the association between these key IncRNAs and TCGA database with the clinicopathological characteristics of the 312 patients 
Table IV. Details information of differentially expressed genes in GEO database.

\begin{tabular}{|c|c|c|c|c|c|}
\hline \multirow{2}{*}{$\begin{array}{l}\text { GSE } \\
\text { GSE23400 }\end{array}$} & \multicolumn{2}{|c|}{$\begin{array}{c}\text { Differentially } \\
\text { expressed genes }\end{array}$} & \multirow{2}{*}{$\begin{array}{c}\text { Upregulated } \\
378\end{array}$} & \multirow{2}{*}{$\frac{\text { Downregulated }}{414}$} & \multirow{2}{*}{$\begin{array}{c}\begin{array}{c}\text { Intersection differentially } \\
\text { expressed genes }\end{array} \\
108\end{array}$} \\
\hline & $\operatorname{lncRNA}$ & 792 & & & \\
\hline GSE26886 & $\operatorname{lncRNA}$ & 1340 & 599 & 741 & \\
\hline GSE45670 & lncRNA & 914 & 508 & 406 & \\
\hline GSE23400 & mRNA & 4518 & 2219 & 2299 & 1234 \\
\hline GSE26886 & mRNA & 7407 & 3933 & 3474 & \\
\hline GSE45670 & mRNA & 5422 & 2599 & 2823 & \\
\hline GSE97049 & miRNA & 178 & 68 & 110 & 45 \\
\hline GSE6188 & miRNA & 198 & 48 & 150 & \\
\hline GSE55856 & miRNA & 219 & 109 & 110 & \\
\hline
\end{tabular}

GEO, Gene Expression Omnibus.
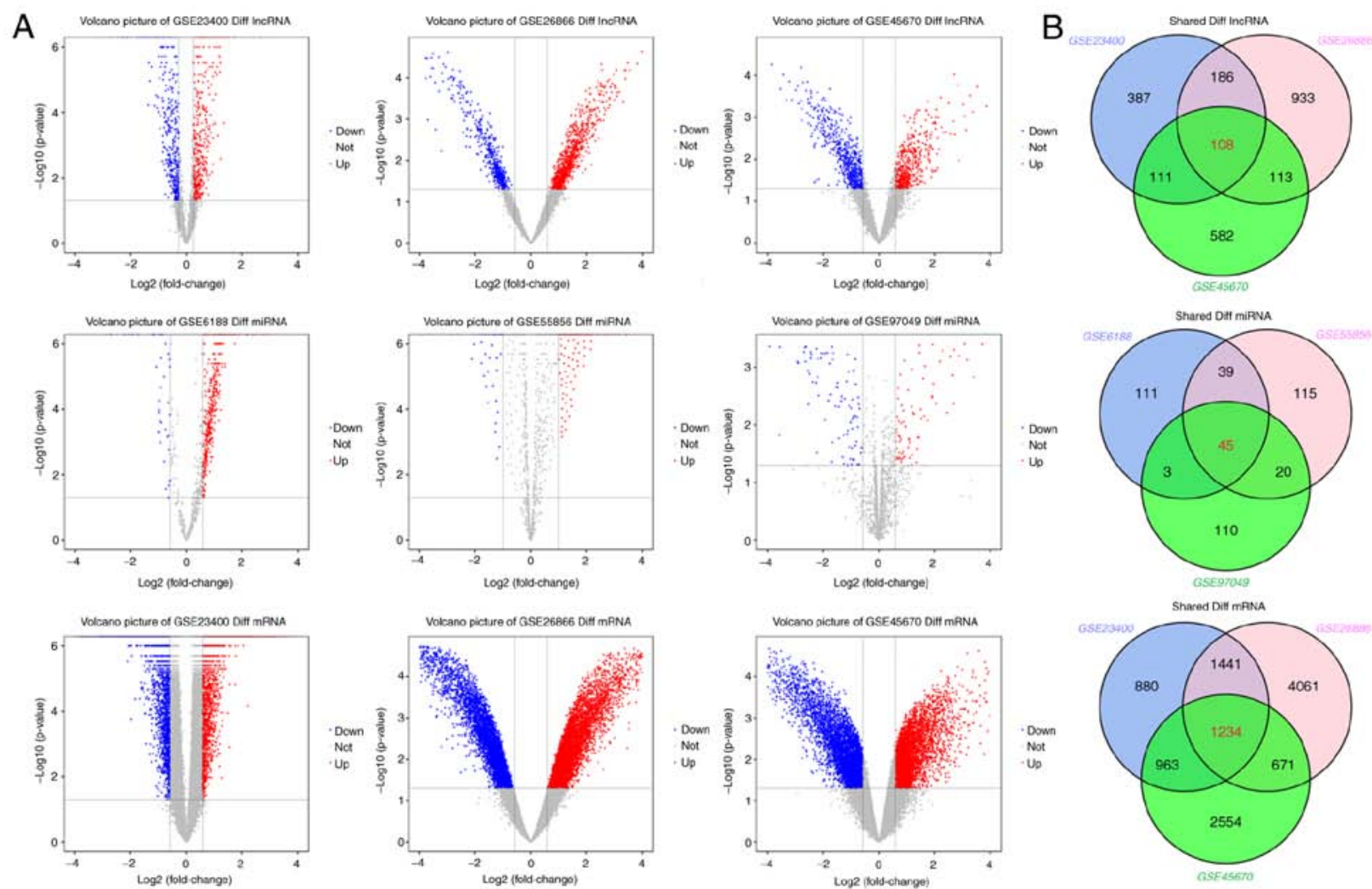

Figure 3. (A) Volcano plots of differentially expressed genes in GEO datasets; (B) Venn diagram demonstrates the differentially expressed intersection genes (lncRNAs, miRNAs and mRNAs) of GEO datasets. GEO, Gene Expression Omnibus; lncRNA, long non-coding RNA.
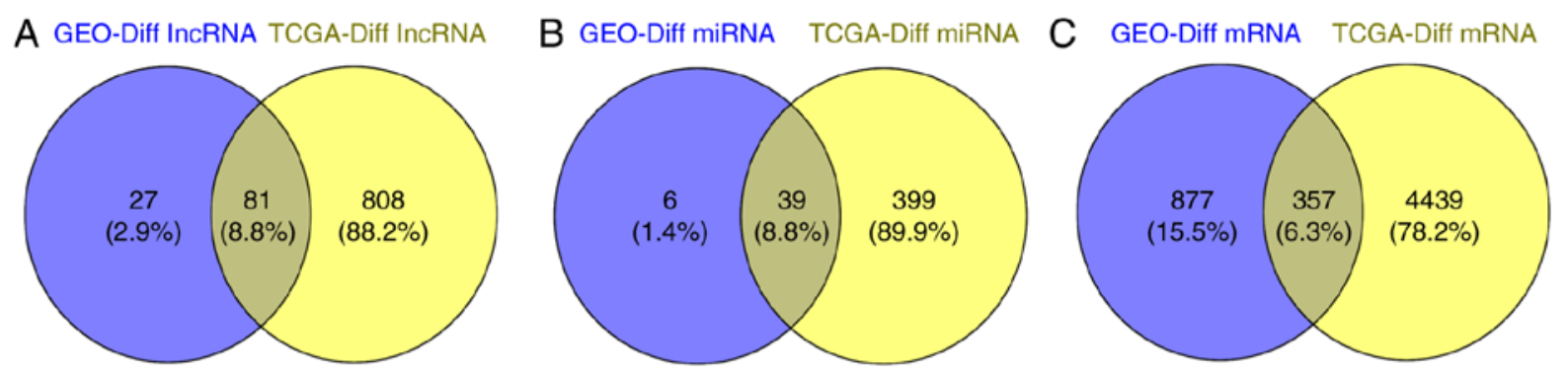

Figure 4. Venn diagram demonstrating the intersections of genes between GEO and TCGA data. (A) The intersection of lncRNAs; (B) The intersection of miRNAs; (C) The intersection of mRNAs. GEO, Gene Expression Omnibus; TCGA, The Cancer Genome Atlas; lncRNA, long non-coding RNA. 
Table V. ESCC-related intersection co-differentially expressed lncRNAs, miRNAs and mRNAs in GEO and TCGA.

Genes

Co-differentially expressed lncRNAs, miRNAs and mRNAs

IncRNAs ALOX12P2, AOC4P, BTN2A3P, C20orf166-AS1, C21orf62-AS1, CMAHP, CYP2D7, CYP4Z2P, DDX12P, DIRC3, DUSP5P1, ENST00000570167.1, ENST00000584492.5, FAM66A, FAM86HP, FAM86JP, FAR2P1, FIRRE, FOXD2-AS1, GUCY1B2, HAND2-AS1, HAVCR1P1, HCP5, HNRNPA3P1, IPW, LINC00176, LINC00341, LINC00346, LINC00472, LINC00476, LINC00663, LINC00689, LINC00887, LINC00889, LINC00950, LINC00982, LINC01001, LINC01105, LINC01588, LOC100128164, LOC100499484-C9ORF174, LOC101928316, LOC148696, LOC202181, LOC283856, LOC399815, LOC728743, MBL1P, MEG3, MIR4435-2HG, MIR503HG, MIR600HG, NONHSAT068116.2, NONHSAT075748.2, NONHSAT179718.1, NONHSAT198787.1, PART1, PCAT18, PSMG3-AS1, PTGES2-AS1, PVT1, PWAR5, PWARSN, RAMP2-AS1, RPLP0P2, SBF1P1, SLC26A4-AS1, SLC8A1-AS1, SMIM10L2A, SMIM10L2B, SNHG4, TCAM1P, TP73-AS1, UCA1, UG0898H09, XR_253656.2, XR_946740.1, ZFAS1, ZFP91-CNTF, ZNF300P1, ZNF542P

miRNAs let-7c-5p, let-7g-3p, miR-101-3p, miR-101-5p, miR-106b-5p, miR-125a-5p, miR-130b-3p, miR-133a-3p, miR-135b-5p, miR-141-3p, miR-143-3p, miR-145-5p, miR-15b-3p, miR-15b-5p, miR-16-5p, miR-182-5p, miR-183-5p, miR-185-5p, miR-18a-5p, miR-195-5p, miR-200a-3p, miR-200b-3p, miR-200c-3p, miR-200c-5p, miR-205-5p, miR-20b-5p, miR-21-3p, miR-224-5p, miR-28-5p, miR-31-5p, miR-320a, miR-32-5p, miR-328-3p, miR-330-5p, miR-33a-5p, miR-425-5p, miR-484, miR-497-5p, miR-93-5p

mRNAs ABCA8, ABCC8, ACACB, ACADL, ACADSB, ACTG2, ACVR2A, ADAMTSL1, ADCY2, ADCY5, ADCY6, ADGRD1, ADH1B, ADHFE1, AFF3, AGPS, ALAD, ALDH6A1, ALDH7A1, ANGPTL1, ANK2, AOX1, APLP1, AQP4, AR, ARHGDIG, ARHGEF6, ARRB1, ASPA, ASXL3, ATP1A2, ATP4A, ATP4B, AZI2, B3GAT1, B4GALNT2, BID, BIRC5, BMP3, BMP8B, BMPER, BMS1, C16orf89, C2orf40, C6, C7, CA4, CAB39L, CACNA2D2, CADM2, CADM3, CALM1, CASQ2, CCBE1, CCKAR, CCKBR, CD1E, CD44, CDC6, CDH19, CDH2, CDK6, CELF4, CFLAR, CGNL1, CHGA, CHGB, CHMP2B, CHRDL1, CHST11, CKB, CKM, CKMT2, CLCNKA, CLDN1, CLDN16, CNKSR2, CNN1, CNTFR, CNTN2, CNTN3, COL2A1, COL4A3, CPA2, CPEB1, CPEB3, CPLX2, CTNND2, CTSC, CUX2, CYBRD1, CYFIP2, CYP2U1, CYP4B1, DES, DHX36, DIRAS1, DLG2, DLG3, DNER, DPP10, DPP6, DPT, E2F2, E2F3, EDA, EDNRB, EFNA5, EIF4EBP2, ELOVL6, EME1, ENAM, ENPP5, EPHA5, EPHB1, ERBB4, ESPL1, ESRRB, ESRRG, ETNPPL, EXO1, FAM107A, FAR1, FAXDC2, FGA, FGF2, FGFR1, FGG, FNDC5, FRMD1, FXYD1, FZD4, GAB1, GAB2, GALNT2, GALNT6, GATA5, GC, GFRA1, GHRL, GIF, GKN1, GKN2, GNAQ, GPD1L, GPER1, GPM6A, GPR155, GREM2, GRIA1, GRIA3, GRIA4, GRIK3, GRIK5, GRIN2A, GSTM5, H2AFJ, H2AFX, HCFC2, HDC, HIPK2, HMGA2, HMGCS2, HMP19, HOXA10, HPN, HPSE2, HS6ST3, HSPB6, HSPB7, ICOS, ID4, IGF1R, IGF2BP1, IKBKE, IL1RAP, IL6ST, INPP5A, IQSEC3, IRS1, ITGA8, ITGB8, ITPR2, KAT2B, KCNB1, KCNE2, KCNJ10, KCNJ11, KCNJ16, KCNK2, KCNMA1, KCNMB2, KCTD8, KIAA0408, KIAA2022, KIF5A, KLF15, KSR1, LAMC2, LAMTOR3, LDB3, LIFR, LIPF, LMOD1, LONRF2, MAGI1, MAGI3, MAMDC2, MAOA, MAP3K13, MAP4K4, MAPK4, MAPT, MARVELD3, MASP1, MFAP5, MFSD4A, MME, MMP14, MOCS1, MT1M, MYH11, MYLK, MYO18B, MYOC, MYOCD, MYRIP, NBEA, NCAM1, NEGR1, NRXN1, NTN4, OAS2, OGN, OMD, P2RX2, PANX1, PCDH9, PCSK2, PDCD4, PDCD6IP, PDE1 A, PDE2A, PDE7B, PDZRN4, PEBP4, PGA3, PGA4, PGA5, PGM5, PGR, PI16, PKHD1L1, PLAU, PLCXD3, PLN, PLP1, PML, PPP1R12B, PPP1R1A, PPP1R9A, PPP2R3A, PRICKLE2, PRIMA1, PRKAA2, PRKACB, PRKAR2B, PRKCB, PRSS1, PSAPL1, PSMB2, PSME4, PTGER3, PTGIS,PTGS1,PTPN2, PTPRN, RAB11A, RAB11FIP2,RAB2A, RAD51, RAG1, RANBP3L, RAP1A, RAPGEF2, RBL1, RBPMS2, RELN, RGN, RIC3, RIMS4, RNF125, RORC, RPRM, RPS6KA2, RPS6KA6, RSPO2, RYR2, S1PR1, SCARA5, SCG3, SCIN, SCN7A, SCUBE2, SEMA3E, SERPINA5, SESN3, SFRP1, SGCA, SH3GL2, SH3GLB1, SIGLEC11, SIX4, SLC1A2, SLC26A7, SLC2A1, SLC2A4, SLC5A7, SLC9A4, SLIT2, SLK, SORCS1, SORT1, SOX10, SOX4, SST, STMN1, STMN2, STUM, SYNPO2, SYT4, TACR1, TCEAL2, TCF3, TFDP2, TGFBR2, THRB, TMEM132C, TNFAIP3, TNFRSF10B, TNXB, TP53INP2, TRA2B, TRIM50, VAMP2, VIP, VIPR2, WASF3, WDR17, WISP2, XKR4, YWHAZ, ZBTB16, ZFP36, ZFP36L2, ZNF385B, ZNF471

ESCC, esophageal squamous cell carcinoma; GEO, Gene Expression Omnibus; TCGA, The Cancer Genome Atlas; lncRNA, long non-coding RNA.

with ESCC. The ESCC patients' clinicopathological characteristics included age, sex, race, tumor grade, TNM stage and lymphatic metastasis, and were obtained from TCGA database.
The relevance analysis results suggested that 31 lncRNAs were significantly differentially expressed in ESCC patients with different clinicopathological characteristics $(\mathrm{P}<0.05)$. 


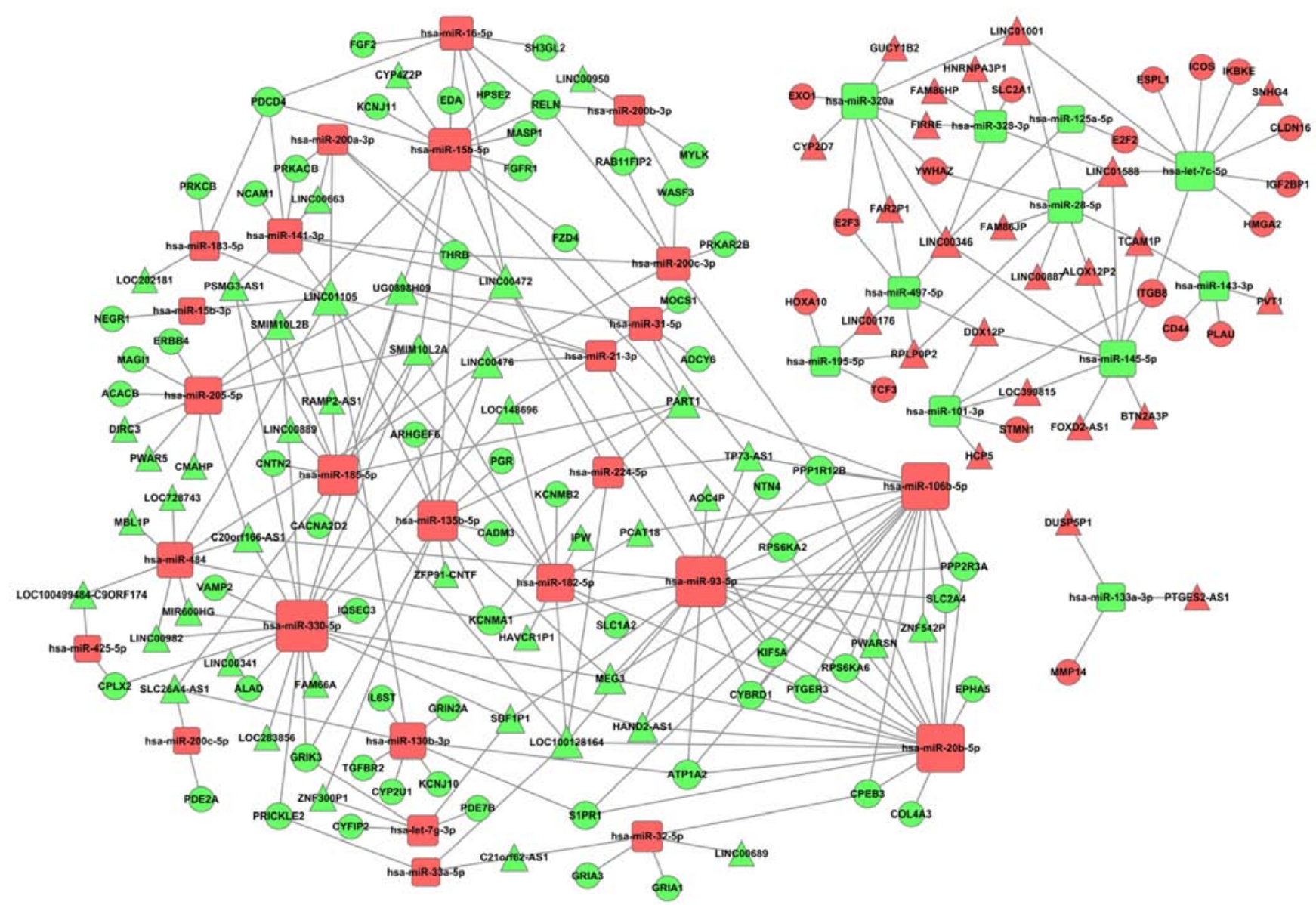

Figure 5. The lncRNA-miRNA-mRNA ceRNA network. Red, upregulated genes; green, downregulated genes; triangles, lncRNAs; squares, miRNAs; balls, mRNAs. lncRNA, long non-coding RNA.

It was revealed that PTGES2-AS1, CMAHP, LINC00472, LINC01105, BTN2A3P, LOC728743, LINC00346, PSMG3-AS1, SBF1P1, LINC01588, DIRC3, RPLPOP2, SMIM10L2A, ZNF300P1 and TP73-AS1 were associated with tumor grade; LOC148696, HCP5, LOC148696, LINC00472, RPLP0P2, DDX12P, SMIM10L2A, PVT1, ZNF300P1, SMIM10L2B and HAND2-AS1 were associated with TNM stage; and PTGES2-AS1, FOXD2-AS1, LOC148696, TCAM1P, LINC00982, LINC00176, DIRC3, ALOX12P2 and SBF1P1 were associated with lymphatic metastasis in patients with ESCC. Furthermore, it was indicated that PART1 and LINC00341 may be associated with sex and race, respectively (Table VI).

Prognostic analysis of IncRNA expression levels and survival of patients with ESCC. Based on the RNA sequencing data and clinical information of the patients with ESCC from TCGA, a Kaplan-Meier survival analysis was used to identify the associations between the ceRNA network 67 key lncRNAs and overall survival time of the ESCC patients. The expression levels of these 67 key lncRNAs and ESCC prognosis data in TCGA database were synthetically calculated using a Cox proportional hazard regression model and it was revealed that there were 15 IncRNAs statistically associated with the overall survival rate (log-rank P<0.05). Among these 15 lncRNAs, 7 (ZNF300P1, TP73-AS1, UG0898H09, CMAHP, DIRC3, LOC148696 and SMIM10L2A) were negatively associated with the prognosis of patients with ESCC $(\mathrm{P}<0.05)$, and 8 (RPLPOP2, HCP5, PVT1, LINC01588, PTGES2-AS1, DDX12P, CYP2D7 and LOC399815) were positively associated with the prognosis of patients with ESCC $(\mathrm{P}<0.05)$ (Fig. 8).

$R T-q P C R$ validation. Through comprehensive analysis of the aforementioned results, it was inferred that LINC00982, TP73-AS1, SMIM10L2A, PVT1 and FOXD2-AS1 may play important roles in ESCC progression. Therefore, these five IncRNAs were selected and their actual expression levels were detected in 30 patients with newly diagnosed ESCC and paired non-tumor esophageal epithelial tissue samples via RT-qPCR to assess the reliability and validity of the bioinformatics analysis results (Tables III and VII). The results revealed that LINC00982, TP73-AS1 and SMIM10L2A were downregulated, whereas PVT1 and FOXD2-AS1 were upregulated in ESCC tumor tissues (Fig. 9). The results suggested that the RT-qPCR validation in 30 newly diagnosed patients with ESCC and the aforementioned bioinformatics analysis results (Table VII) exhibited the same trends (Fig. 5).

Subsequently, the association between the real expression levels of the aforementioned 5 lncRNAs and the clinical characteristics of the 30 newly diagnosed patients with ESCC was assessed. The results revealed that TP73-AS1, SMIM10L2A and PVT1 were significantly associated with TNM stage $(\mathrm{P}<0.05)$, whereas LINC00982, SMIM10L2A and FOXD2-AS1 were significantly associated with lymph node 
A

Difgene Sig GO

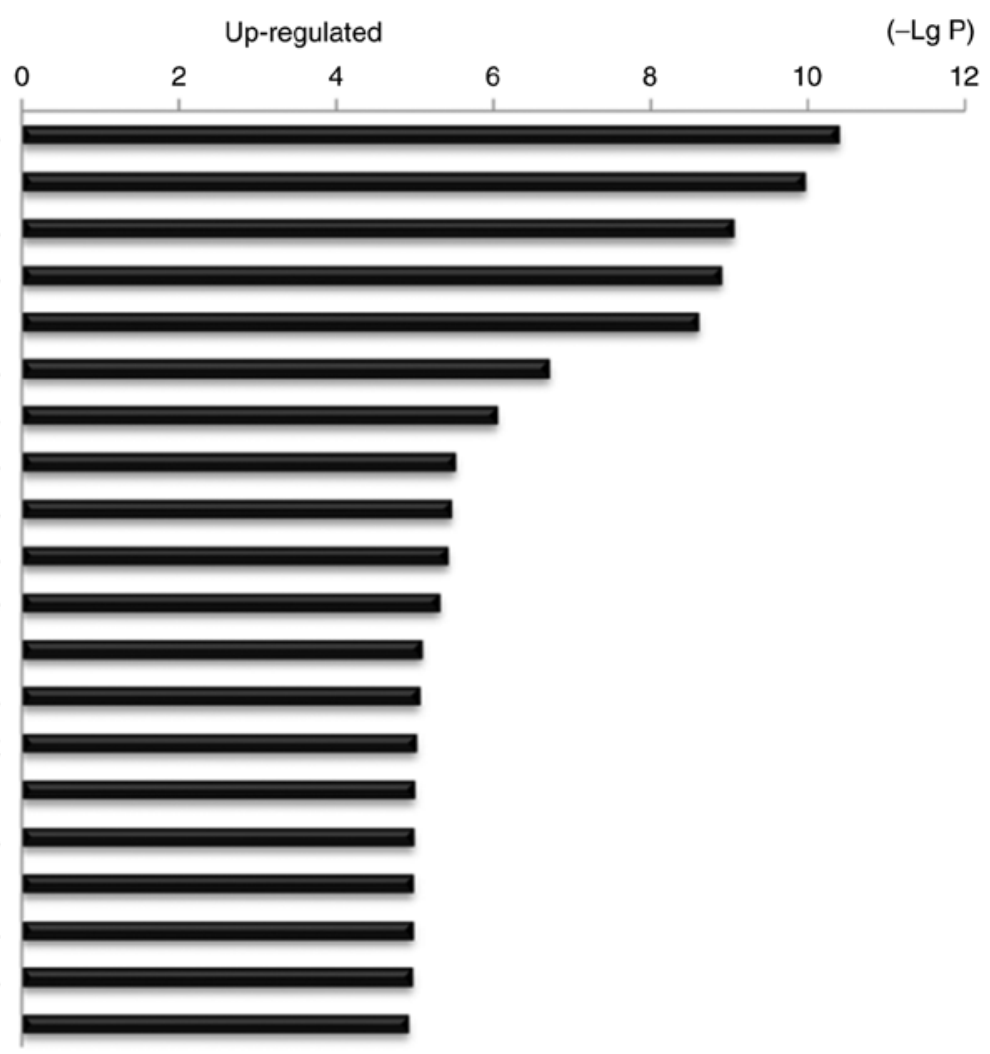

B

Difgene Sig GO

Down-regulated

$(-\operatorname{Lg} P)$

Cellular response to stress

Transcription factor complex

Signaling

Regulation of metabolic process

Cell communication

Single organism reproductive process

Response to stress

Cell death

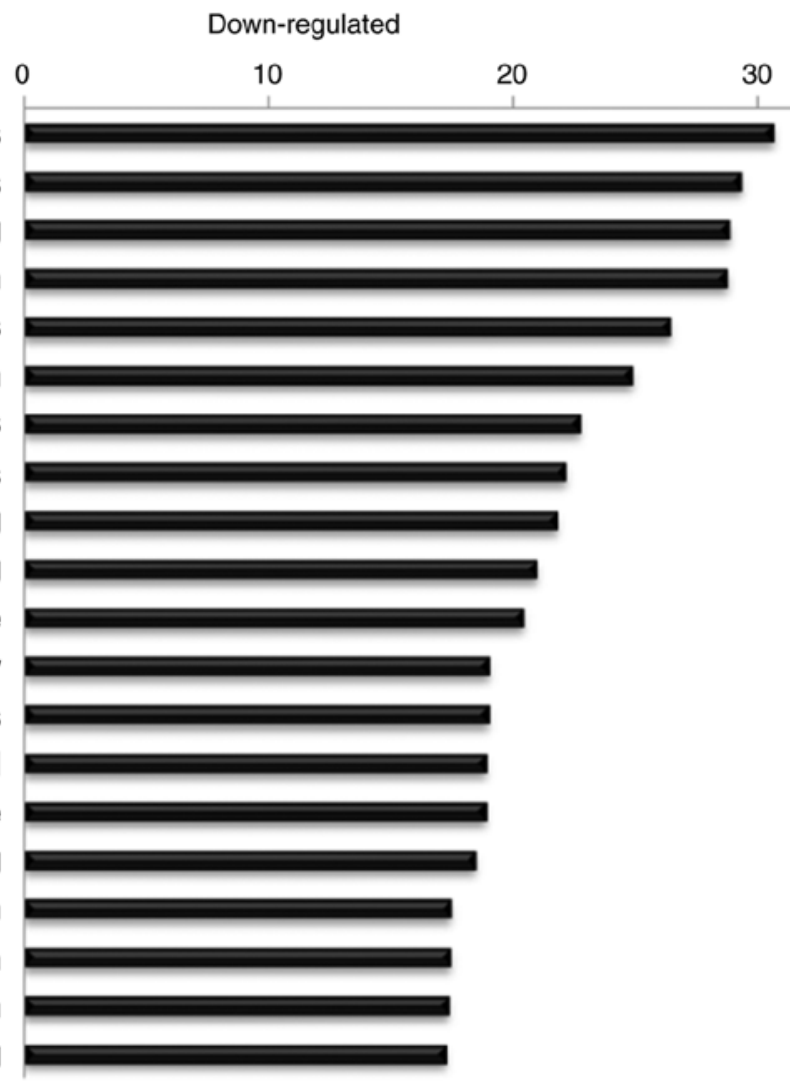

Single-organism cellular process

Response to stimulus

Signaling

Cell communication

Cellular process

Biological regulation

Cellular response to stimulus

Regulation of biological process

Cell-cell signaling

Binding

Plasma membrane

Regulation of biological quality

System process

Response to chemical

Membrane

Protein binding

Regulation of cell communication

Cell projection

Signal transduction

Regulation of signaling

Figure 6. Top 20 enrichment of GO terms for mRNAs in the ceRNA network (the bar plot shows the enrichment scores of the significant enrichment GO terms). GO, Gene Ontology.

metastasis status $(\mathrm{P}<0.05)$. In addition, it was also revealed that TP73-AS1, SMIM10L2A and FOXD2-AS1 were correlated with the age and sex of the patients with $\operatorname{ESCC}(\mathrm{P}<0.05$; Fig. 10). The expression levels of the 5 IncRNAs were detected, 
A

Difgene Sig pathway

Up-regulated

(-Lg P)

A

MicroRNAs in cancer

Transcriptional misregulation in cancer

Cell adhesion molecules (CAMs)

Bladder cancer

Non-small cell lung cancer

Wnt signaling pathway

MAPK signaling pathway

Melanoma

Chronic myeloid leukemia

ECM-receptor interaction

Proteoglycans in cancer

PI3K-Akt signaling pathway

Mismatch repair

Primary immunodeficiency

TNF signaling pathway

Thyroid hormone signaling pathway

Leukocyte transendothelial migration

Measles

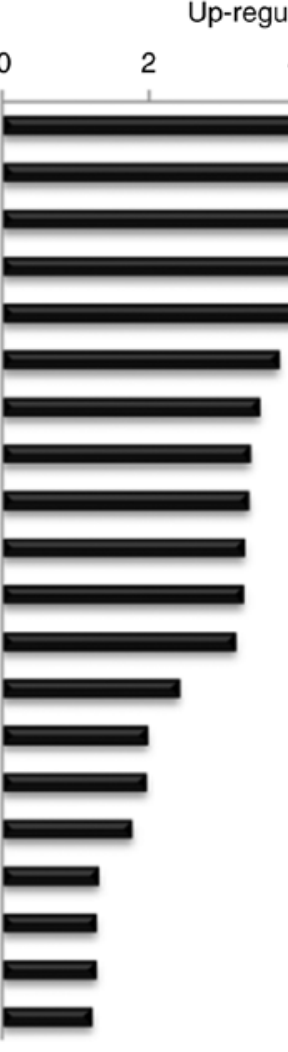

B

Difgene Sig pathway

Down-regulated

$(-\operatorname{Lg} P)$

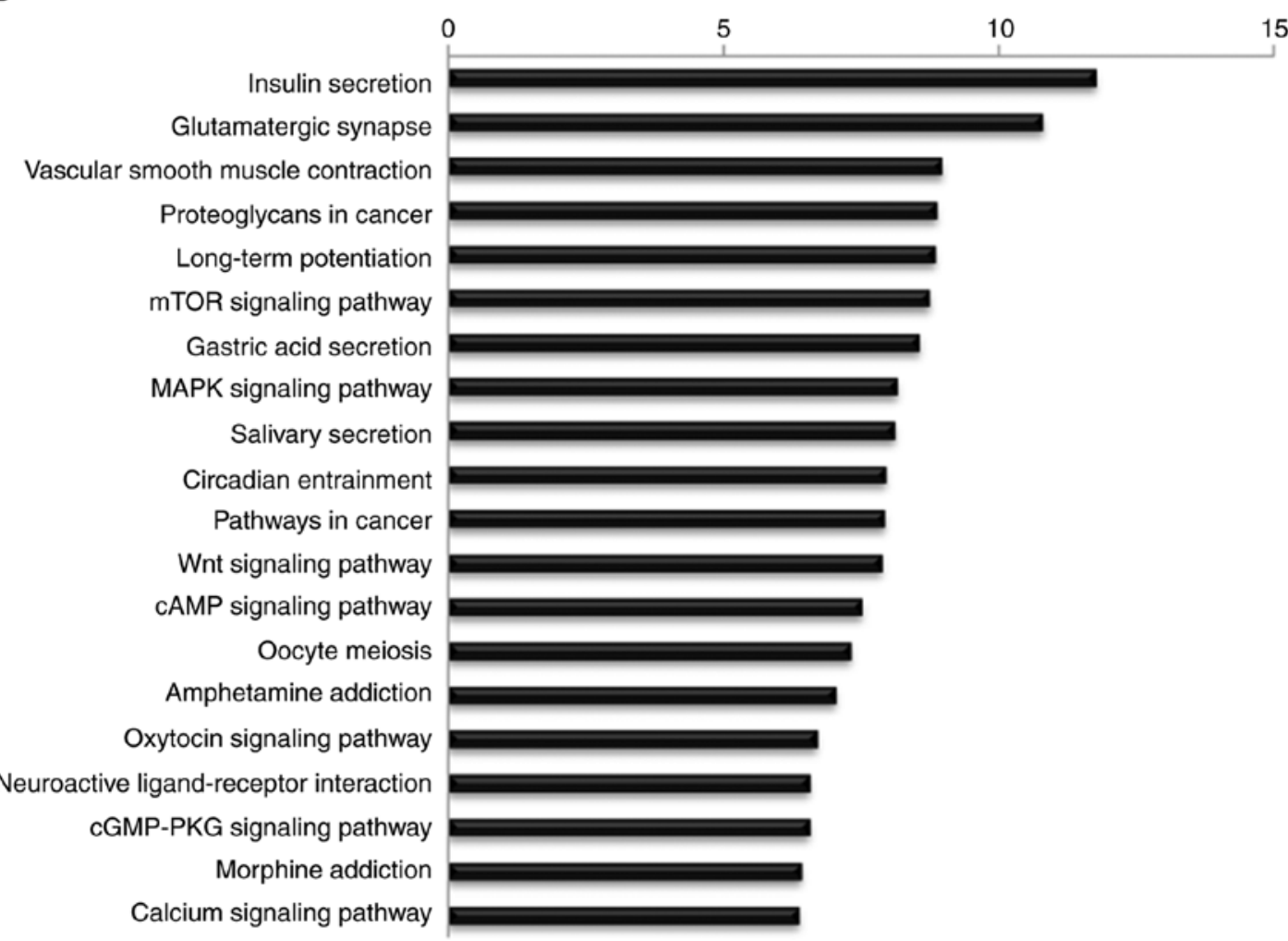

Figure 7. Top 20 enrichment of pathways for mRNAs in ceRNA network (the bar plot shows the enrichment scores of the significant enrichment pathways).

and the results from the clinically relevant analysis and the aforementioned bioinformatics analysis (Tables V and VII) were similar, suggesting that the bioinformatics analysis used in the present study was credible.

\section{Discussion}

ESCC is a common malignant tumor originating from the mucosa of the esophagus at vulnerable sites, including the 
Table VI. Associations between lncRNA signature and ESCC patients' clinicopathological characteristics.

\begin{tabular}{lll}
\hline Comparisons & Upregulated & Downregulated
\end{tabular}

Sex (male vs. female)

Race

(Caucasian vs. Asian)

Tumor grade

(GIII-IV vs. GI-II)

TNM stage

$(\mathrm{T} 3+\mathrm{T} 4$ vs. $\mathrm{T} 1+\mathrm{T} 2)$

Lymphatic metastasis

(yes vs. no)
ALOX12P2, DUSP5P1

PTGES2-AS1, LINC01588, BTN2A3P, LOC728743, RPLPOP2, LINC00346 RPLP0P2, DDX12P, PVT1, HCP5

PTGES2-AS1, FOXD2-AS1, TCAM1P, LINC00176

\author{
C20orf166-AS1, UG0898H09, PART1 \\ PART1, LINC00341, LINC00982, DIRC3
}

PSMG3-AS1, CMAHP, LINC00472, LINC01105, SBF1P1, DIRC3, SMIM10L2A, ZNF300P1, TP73-AS1 LINC00472, LOC148696, SMIM10L2A, ZNF300P1, SMIM10L2B, HAND2-AS 1

DIRC3, LINC00982, LOC148696, SBF1P1

ESCC, esophageal squamous cell carcinoma; lncRNA, long non-coding RNA.
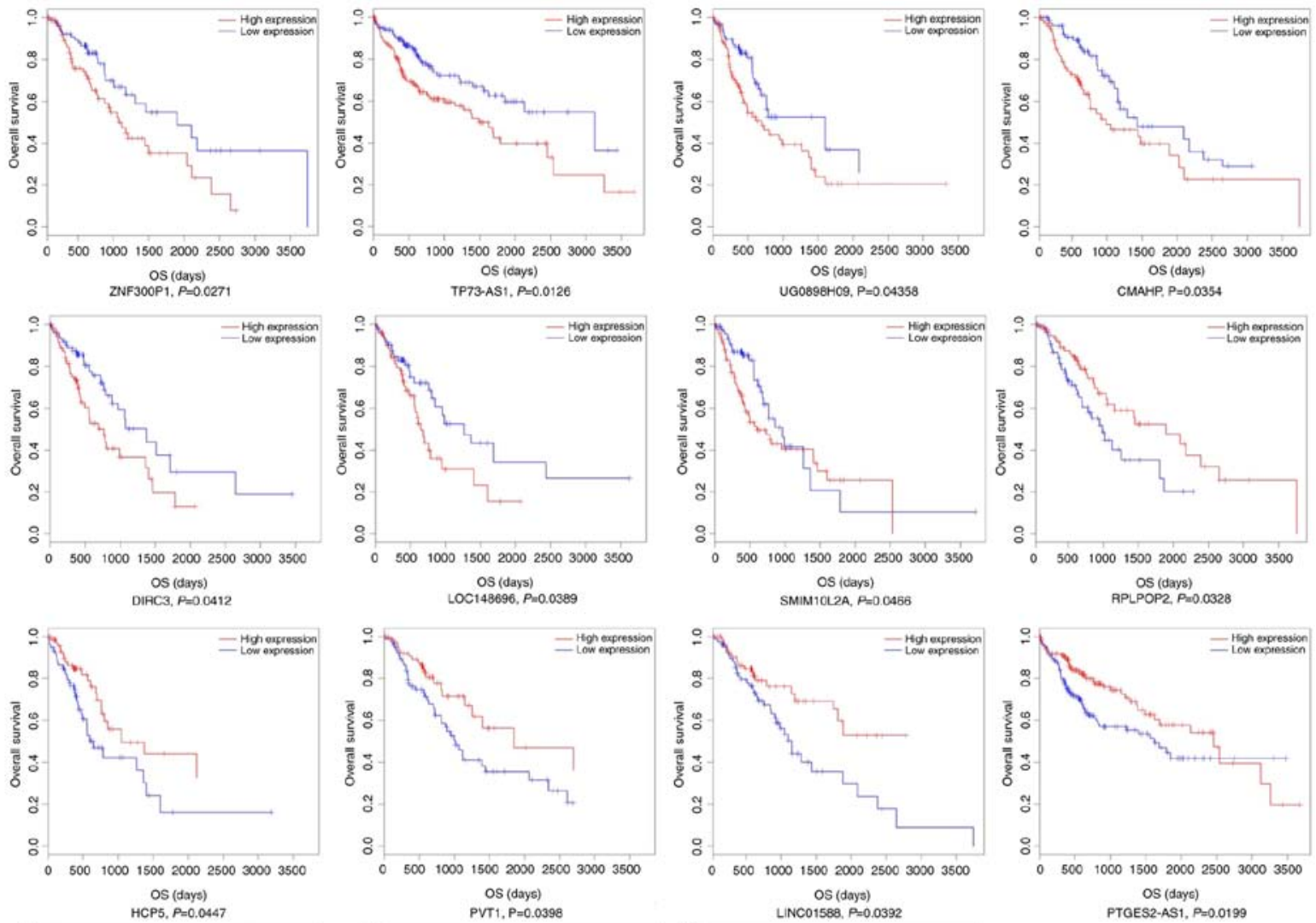

OS (days)
RPLPOP2 P
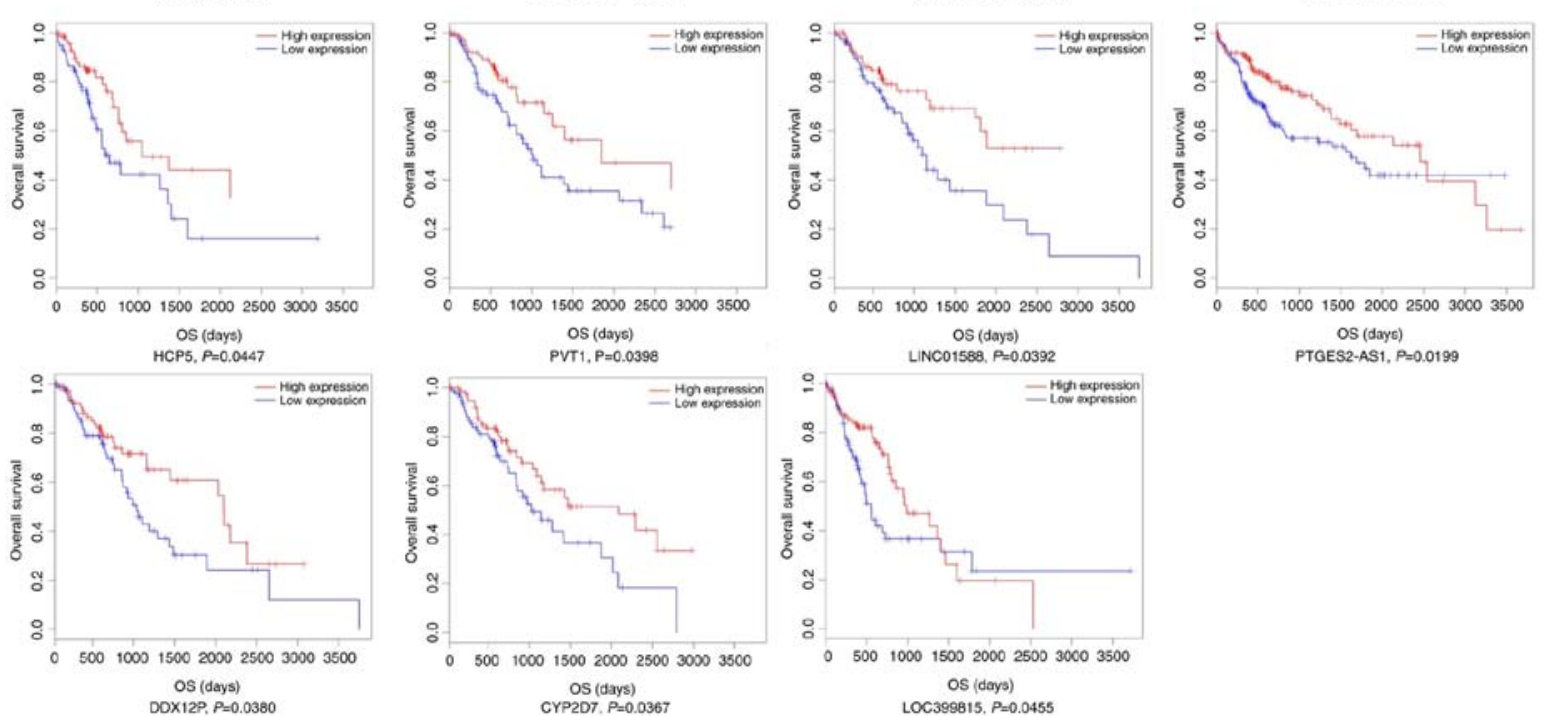

Figure 8. Kaplan-Meier survival curves for 15 lncRNAs associated with ESCC patients' overall survival time [horizontal axis, overall survival time (days); vertical axis, survival function]. ESCC, esophageal squamous cell carcinoma; lcRNA, long non-coding RNA.

pharyngoesophageal junction, the part of the esophagus crossing with the posterior surface of the left bronchus, and the part of the esophagus passing through the diaphragmatic esophageal hiatus (25). Gastrointestinal endoscopy detection cannot identify all precancerous conditions or the early stages of ESCC. The incidence and mortality rate of ESCC in the 
Table VII. Randomly selected lncRNAs with absolute $\mathrm{FC}>2, \mathrm{P}<0.05$.

\begin{tabular}{lrcrr}
\hline Name (lncRNAs) & Gene ID & Regulation & TCGA (mean FC) & GEO (mean FC) \\
\hline LINC00982 & 440556 & Down & -17.539 & -3.991 \\
TP73-AS1 & 57212 & Down & -3.845 & -5.579 \\
SMIM10L2A & 399668 & Down & -7.850 & -4.852 \\
PVT1 & 5820 & Up & 7.193 & 9.997 \\
FOXD2-AS1 & 84793 & Up & 6.470 & 14.211 \\
\hline
\end{tabular}

FC, fold change; TCGA, The Cancer Genome Atlas; GEO, Gene Expression Omnibus; lncRNA, long non-coding RNA.

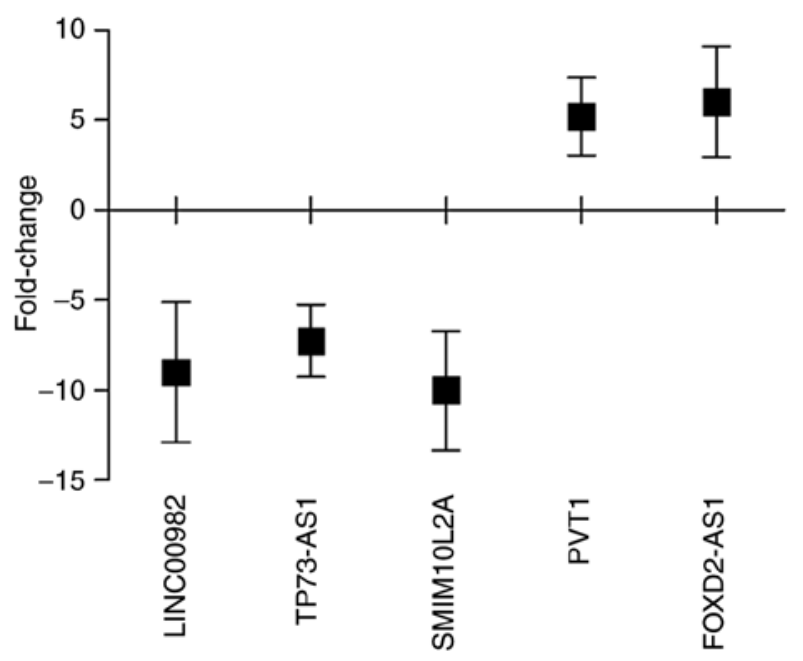

Figure 9. Box plot showing the median and quartiles of specific lncRNAs in donor samples. IncRNA, long non-coding RNA.

general population are high, and a large number of patients are usually diagnosed late and have a poor prognosis (26). In addition, ESCC lymph node metastasis may be regional, bidirectional, continuous or jumping, and effective complete eradication methods are currently lacking (27). In order to improve survival rate and the detection and treatment of early-stage ESCC, more effective therapeutic tools are required, including the identification of novel diagnostic and prognostic biomarkers. Recent studies that have focused on the abnormal expression of lncRNAs have revealed a new investigative approach to the pathological changes observed in ESCC, and have also indicated that the search for novel biomarkers may hold promise for ESCC diagnosis and prognosis (28-30). The main concern is that small amounts of RNA sequencing samples may not accurately reflect the abnormal changes in IncRNAs when used as biomarkers that are associated with the diagnosis and prognosis of $\operatorname{ESCC}(31,32)$. Therefore, the ESCC-associated dysregulated lncRNA expression profiles should be identified based on large sample sizes in order to improve their accuracy and reliability as diagnostic and prognostic markers.

The development of high throughput RNA sequencing technologies has allowed thousands of dysregulated RNAs to be observed in a number of diseases (33-36). The present study identified abnormally expressed lncRNAs implicated in the pathogenesis of ESCC by synthetically analyzing RNA sequencing datasets obtained from the GEO and TCGA databases. A number of studies have proved that integration of multiple RNA sequencing datasets is a better method for enhancing the accuracy and reliability of results compared with using individual small samples (37). RNA sequencing allows reannotation, identification of differentially expressed RNAs in the GEO and TCGA databases, ceRNA network construction based on gene discovery, association between key lncRNAs and clinical characteristics of ESCC patients and survival analysis, in order to investigate the potential biomarkers for the diagnosis and prognosis of ESCC.

A multiple subset analysis and integrated bioinformatics approach was applied in order to identify dysregulated RNAs in ESCC in the present study. In order to increase the gene information accuracy, the RNA sequencing signals from the GEO database were matched to their chromosomal position using the GENCODE tool. All dataset samples were used in order to increase the sample numbers so as to avoid errors in the results and narrow the gene objects range. In addition, to avoid errors occurring from different ESCC individual tissues, only paired RNA sequencing sample datasets were included in the GEO database.

According to the integrated bioinformatics approach, it was revealed that there were 81 lncRNAs, 39 miRNAs and 357 mRNAs co-differentially expressed ESCC-associated intersection genes between the GEO and TCGA database. Among these 81 dysregulated lncRNAs, some have been indicated to exhibit significantly different expression levels in ESCC, including downregulated lncRNA HAND2-AS1 which may act as an anti-oncogene that can inhibit ESCC cell proliferation, migration and invasion (38). Dong et al (39) and Huang et al (40) have reported that downregulated MEG3 in ESCC tissues can inhibit ESCC cell growth and induce apoptosis. In addition, upregulated lncRNAs PVT1, TP73-AS1, UCA1 and ZFAS1 were also reported to be significantly differentially expressed in esophageal cancer, and also to be involved in regulating the progression of this disease (41-44). To the best of our knowledge, the functions of the remaining dysregulated lncRNAs in the development and progression of ESCC have not yet been reported.

Based on the theory that lncRNAs can regulate miRNA abundance by sequestration binding, acting as 'miRNA sponges', and the fact that miRNAs can bind to mRNAs and negatively regulate gene expression (45), the present study built an IncRNA-miRNA-mRNA ceRNA network according to their 

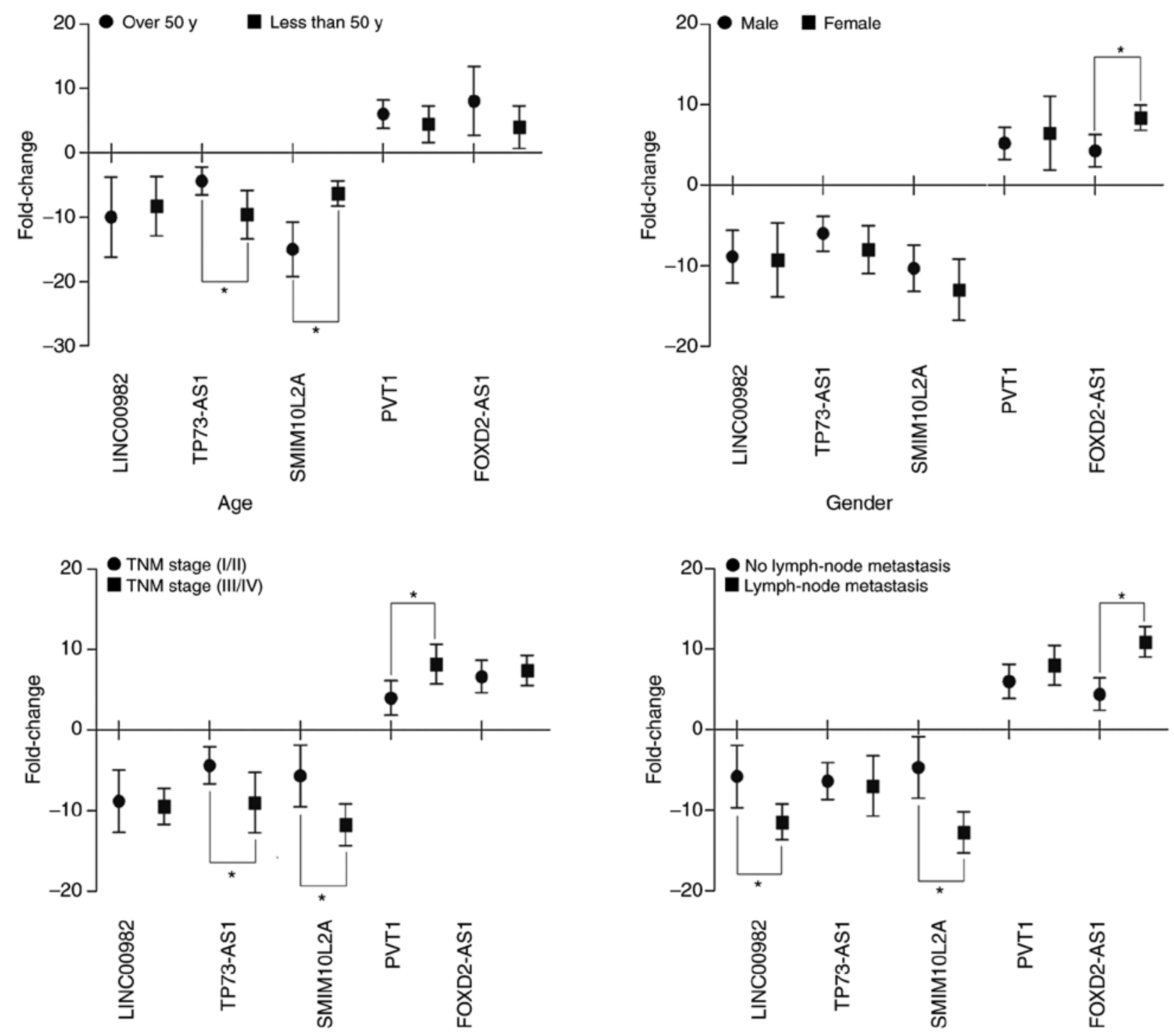

TNM staging
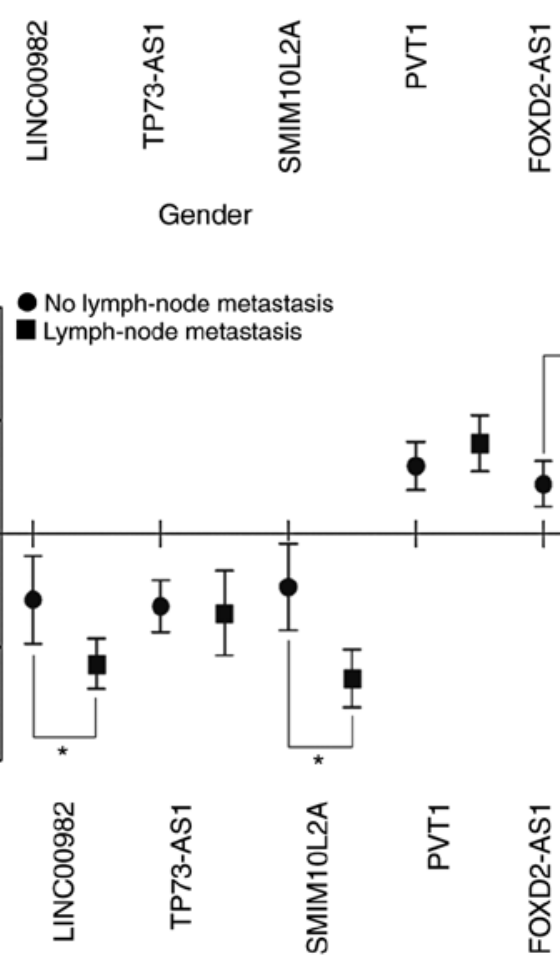

Lymph-node metastasis status

Figure 10. Box plot showing the association of the fold change in LINC00982, TP73-AS1, SMIM10L2A, PVT1 and FOXD2-AS1 expression with clinicopathological characteristics in 30 ESCC patients. ESCC, esophageal squamous cell carcinoma; y, years. ${ }^{*} \mathrm{P}<0.05$.

negative regulatory associations. Among 81 ESCC-associated intersection lncRNAs, a total of 67 key lncRNAs were included in the IncRNA-miRNA-mRNA ceRNA network. The ceRNA network construction can reveal the potential regulatory associations between lncRNAs, miRNAs and mRNAs in ESCC. According to the ceRNA network created in the present study, some of these 67 lncRNAs, such as FIRRE, FOXD2-AS1, IPW and LINC00472, were also reported as potential diagnostic and prognostic biomarkers in numerous human diseases, including ESCC (46-49). In order to investigate the potential regulatory functions of these 67 key lncRNAs, the present study further analyzed the 80 negatively regulated mRNAs in the ceRNA network, and revealed that some of those also play important roles in the progression of ESCC, including CYBRD1, EDA, ERBB4, FGF2 and IKBKE (50-52). Subsequently, the present study analyzed the 67 key lncRNAs in the ceRNA network and identified enrichment of the functions of 80 mRNAs that were indirectly involved in signaling pathways. The GO analysis results revealed that the enriched GOs primarily targeted the metabolism and cell biological process. The KEGG analysis revealed that certain pathways were also associated with cancer, such as the Wnt signaling pathway, the MAPK signaling pathway, non-small cell lung cancer, pathways in cancer and the PI3K-AKT signaling pathway (53). Therefore, the present bioinformatics analysis results revealed that these 67 key lncRNAs in the ceRNA network may be implicated in the progression of ESCC.

The associations between the expression levels of the 67 aforementioned key lncRNAs and the clinicopathological characteristics from TCGA database were analyzed, and it was revealed that 32 lncRNAs were associated with the characteristics of the 312 patients with ESCC. These lncRNAs were primarily associated with tumor grade, TNM stage and lymphatic metastasis status. Among these 31 lncRNAs, PVT1, FOXD2-AS1 and PART1 have been reported to be the key genes involved in lymphatic metastasis and invasion and as diagnostic biomarkers for ESCC (54-56). However, the functions of other IncRNAs have not yet been reported 
in association with ESCC progression. Therefore, the present study also analyzed the association between the 67 key lncRNAs in the ceRNA network and overall survival of patients with ESCC in TCGA database. It was revealed that 15 lncRNAs were associated with overall survival time. Among these 15 lncRNAs, only TP73-AS1 and PVT1 have been reported to be associated with survival in ESCC $(42,57)$, whereas other IncRNAs have not been reported to date. The bioinformatics analysis results in the present study revealed potential lncRNAs biomarkers for the diagnosis, classification and prognosis of ESCC.

Subsequently, RT-qPCR validation of 5 randomly selected lncRNAs in 30 ESCC tissue samples was performed and the accuracy and credibility of the bioinformatics results were assessed. The results of the RT-qPCR validation were almost the same as the expression data in the GEO and TCGA databases. The present study then analyzed the association between these 5 randomly selected lncRNAs and the collected clinicopathological data of the patients with ESCC. The results also suggested that the results with these 5 lncRNAs were similar to the aforementioned bioinformatics analysis results. Therefore, the synthetic bioinformatics analysis results of the present study are reliable.

In summary, the present study has successfully identified specific ESCC-associated lncRNAs from large-scale samples through integrated analysis of RNA expression profile datasets of patients with ESCC from the GEO and TCGA databases. Differentially expressed lncRNAs and their potential functions in ESCC were investigated in the present study, along with specific ESCC-associated lncRNAs by different clinicopathological characteristics and overall survival time of patients with ESCC. Overall, these 67 lncRNAs are worth investigating further in terms of their applicability as biomarkers in the diagnosis, clinicopathological classification and prognosis of patients with ESCC.

\section{Acknowledgements}

Not applicable.

\section{Funding}

The present study was supported by the Fundamental Research Funds for the Central Universities (grant no. lzujbky-2018-13), the National Natural Science Foundation of China (grant no. 81803188), and the Gansu Province Science and Technology Project (grant no. 1606RJYA270).

\section{Availability of data and materials}

The authors declare that all relevant raw data will be made freely available to any researchers who wish to use them for non-commercial purposes, whilst preserving any necessary confidentiality and anonymity. All data generated or analyzed during this study are included in this published article.

\section{Authors' contributions}

CYL conceived and designed the study. CYL and XHW performed the experiments. WWZ, JLW and JL analyzed and interpreted the results. JLX and XHW performed the patient tissue sample collection and quality control. CYL performed analysis and quality control, and was a major contributor to writing the manuscript. All authors have read and approved the final manuscript.

\section{Ethics approval and consent to participate}

The present study was approved by the Ethics Committee of the Gansu Wuwei Tumor Hospital. All patients provided written informed consent to participate in the present study.

\section{Patient consent for publication}

Not applicable.

\section{Competing interests}

The authors declare that they have no competing interests.

\section{References}

1. Bray F, Ferlay J, Soerjomataram I, Siegel RL, Torre LA and Jemal A: Global cancer statistics 2018: GLOBOCAN estimates of incidence and mortality worldwide for 36 cancers in 185 countries. CA Cancer J Clin 68: 394-424, 2018.

2. Jiang $\mathrm{C}$, Chen $\mathrm{Y}, \mathrm{Zhu} \mathrm{Y}$ and $\mathrm{Xu} \mathrm{Y}$ : Systematic review and meta-analysis of the accuracy of 18F-FDG PET/CT for detection of regional lymph node metastasis in esophageal squamous cell carcinoma. J Thorac Dis 10: 6066-6076, 2018.

3. Kim M: Evaluation of a web-based App demonstrating an exclusionary algorithmic approach to TNM cancer staging. JMIR Cancer 1: e3, 2015.

4. Zhang X, Wang Y, Zhao L, Sang S and Zhang L: Prognostic value of platelet-to-lymphocyte ratio in oncologic outcomes of esophageal cancer: A systematic review and meta-analysis. Int J Biol Markers: Apr 1, 2018 (Epub ahead of print).

5. Quan J, Pan X, Zhao L, Li Z, Dai K, Yan F, Liu S, Ma H and Lai Y: LncRNA as a diagnostic and prognostic biomarker in bladder cancer: A systematic review and meta-analysis. Onco Targets Ther 11: 6415-6424, 2018.

6. Tian T, Wang M, Lin S, Guo Y, Dai Z, Liu K, Yang P, Dai C, Zhu Y, Zheng Y, et al: The impact of lncRNA dysregulation on clinicopathology and survival of breast cancer: A systematic review and meta-analysis. Mol Ther Nucleic Acids 12: 359-369, 2018.

7. Liu W, Zhang Y, Chen M, Shi L, Xu L and Zou X: A genome-wide analysis of long noncoding RNA profile identifies differentially expressed lncRNAs associated with Esophageal cancer. Cancer Med 7: 4181-4189, 2018.

8. Khadirnaikar S, Narayanan SP and Shukla SK: Decoding the LncRNA transcriptome of esophageal cancer: Identification of clinically relevant LncRNAs. Biomark Med 12: 1083-1093, 2018.

9. Ji S, Yang W, Liu J, Zhao J, Chen L, Ni Q, Long J and Yu X: High throughput gene sequencing reveals altered landscape in DNA damage responses and chromatin remodeling in sporadic pancreatic neuroendocrine tumors. Pancreatology 18: 318-327, 2018.

10. Krishnamurthy A, Ferl RJ and Paul AL: Comparing RNA-Seq and microarray gene expression data in two zones of the Arabidopsis root apex relevant to spaceflight. Appl Plant Sci 6: e01197, 2018.

11. Hu J, Zhou L, Song Z, Xiong M, Zhang Y, Yang Y, Chen K and Chen Z: The identification of new biomarkers for bladder cancer: A study based on TCGA and GEO datasets. J Cell Physiol, Feb 18, 2019 (Epub ahead of print).

12. Su H, Hu N, Yang HH, Wang C, Takikita M, Wang QH, Giffen C, Clifford R, Hewitt SM, Shou JZ, et al: Global gene expression profiling and validation in esophageal squamous cell carcinoma and its association with clinical phenotypes. Clin Cancer Res 17: 2955-2966, 2011. 
13. Wang Q, Ma C and Kemmner W: Wdr66 is a novel marker for risk stratification and involved in epithelial-mesenchymal transition of esophageal squamous cell carcinoma. BMC Cancer 13: 137, 2013

14. Wen J, Yang H, Liu MZ, Luo KJ, Liu H, Hu Y, Zhang X, Lai RC, Lin T, Wang HY and Fu JH: Gene expression analysis of pretreatment biopsies predicts the pathological response of esophageal squamous cell carcinomas to neo-chemoradiotherapy. Ann Oncol 25: 1769-1774, 2014

15. Guo Y, Chen Z, Zhang L, Zhou F, Shi S, Feng X, Li B, Meng X, Ma X, Luo M, et al: Distinctive microRNA profiles relating to patient survival in esophageal squamous cell carcinoma. Cancer Res 68: 26-33, 2008.

16. Jang HJ, Lee HS, Burt BM, Lee GK, Yoon KA, Park YY, Sohn BH, Kim SB, Kim MS, Lee JM, et al: Integrated genomic analysis of recurrence-associated small non-coding RNAs in oesophageal cancer. Gut 66: 215-225, 2017

17. Livak KJ and Schmittgen TD: Analysis of relative gene expression data using real-time quantitative PCR and the 2(-Delta Delta C(T)) Method. Methods 25: 402-408, 2001.

18. Cao B, Yang W, Jin Y, Zhang M, He T, Zhan Q, Herman JG, Zhong $\mathrm{G}$ and Guo M: Silencing NKD2 by promoter region hypermethylation promotes esophageal cancer progression by activating Wnt signaling. J Thorac Oncol 11: 1912-1926, 2016.

19. Wang X, Zhu Y, Zhu L, Chen X, Xu Y, Zhao Y, Shao Y, Li F Jiang Y, Lu J, et al: Eupatilin inhibits the proliferation of human esophageal cancer TE1 cells by targeting the Akt-GSK3 $\beta$ and MAPK/ERK signaling cascades. Oncol Rep 39: 2942-2950, 2018.

20. Jiang JH, Pi J, Jin H and Cai JY: Oridonin-induced mitochondria-dependent apoptosis in esophageal cancer cells by inhibiting PI3K/AKT/mTOR and Ras/Raf pathways. J Cell Biochem 120: 3736-3746, 2019.

21. He J, Zhao J, Zhu W, Qi D, Wang L, Sun J, Wang B, Ma X, Dai Q and $\mathrm{Yu} \mathrm{X}$ : MicroRNA biogenesis pathway genes polymorphisms and cancer risk: A systematic review and meta-analysis. PeerJ 4: e2706, 2016.

22. Hu M, Zhu S, Xiong S, Xue X and Zhou X: MicroRNAs and the PTEN/PI3K/Akt pathway in gastric cancer (Review). Oncol Rep 41: 1439-1454, 2019.

23. Xiao YF, Yong X, Tang B, Qin Y, Zhang JW, Zhang D, Xie R and Yang SM: Notch and Wnt signaling pathway in cancer: Crucial role and potential therapeutic targets (Review). Int J Oncol 48 437-449, 2016

24. Toren P and Zoubeidi A: Targeting the PI3K/Akt pathway in prostate cancer: Challenges and opportunities (Review). Int $\mathbf{J}$ Oncol 45: 1793-1801, 2014.

25. Hayashi Y, Nishida T, Tsujii M, Tsutsui S, Yamamoto K, Isohashi $\mathrm{F}$, Yamasaki M, Miyata $\mathrm{H}$, Kato M, Yamada T, et al: Lymph node enlargement after definitive chemoradiotherapy for clinical stage I esophageal squamous cell carcinoma. BMC Cancer 14: 706, 2014.

26. Cheng YF, Chen HS, Wu SC, Chen HC, Hung WH, Lin CH and Wang BY: Esophageal squamous cell carcinoma and prognosis in Taiwan. Cancer Med 7: 4193-4201, 2018

27. Mine S, Watanabe M, Kumagai K, Okamura A, Yuda M, Hayami M, Yamashita K, Imamura Y and Ishizuka N: Comparison of mediastinal lymph node metastases from adenocarcinoma of the esophagogastric junction versus lower esophageal squamous cell carcinoma with involvement of the esophagogastric junction. Dis Esophagus: Feb 22, 2019 (Epub ahead of print).

28. Huang GW, Xue YJ, Wu ZY, Xu XE, Wu JY, Cao HH, Zhu Y, He JZ, Li CQ, Li EM and Xu LY: A three-lncRNA signature predicts overall survival and disease-free survival in patients with esophageal squamous cell carcinoma. BMC Cancer 18: 147, 2018.

29. Wang J, Sun D, Wu K, Liu J, Zhao M, Li X, Xu Y and Li B: Genome-wide analysis of long non-coding RNAs in esophageal squamous cell carcinoma reveals their potential role in invasion and metastasis. Thorac Cancer 10: 78-89, 2019.

30. Alaei S, Sadeghi B, Najafi A and Masoudi-Nejad A: LncRNA and mRNA integration network reconstruction reveals novel key regulators in esophageal squamous-cell carcinoma. Genomics 111: 76-89, 2019.

31. Campbell JD, Liu G, Luo L, Xiao J, Gerrein J, Juan-Guardela B, Tedrow J, Alekseyev YO, Yang IV, Correll M, et al: Assessment of microRNA differential expression and detection in multiplexed small RNA sequencing data. RNA 21: 164-171, 2015.

32. Collins JE, Wali N, Sealy IM, Morris JA, White RJ, Leonard SR, Jackson DK, Jones MC, Smerdon NC, Zamora J, et al: High-throughput and quantitative genome-wide messenger RNA sequencing for molecular phenotyping. BMC Genomics 16: 578 , 2015 .
33. Ozsolak F: Attomole-level genomics with single-molecule direct DNA, cDNA and RNA sequencing technologies. Curr Issues Mol Biol 18: 43-48, 2016.

34. Lacaze É, Gendron AD, Miller JL, Colson TL, Sherry JP, Giraudo M, Marcogliese DJ and Houde M: Cumulative effects of municipal effluent and parasite infection in yellow perch: A field study using high-throughput RNA-sequencing. Sci Total Environ 665: 797-809, 2019

35. Ge L, Liu S, Xie L, Sang L, Ma C and Li H: Differential mRNA expression profiling of oral squamous cell carcinoma by high-throughput RNA sequencing. J Biomed Res 29, 2015 (Epub ahead of print).

36. Gao M, Zhong A, Patel N, Alur C and Vyas D: High throughput RNA sequencing utility for diagnosis and prognosis in colon diseases. World J Gastroenterol 23: 2819-2825, 2017.

37. Ma T, Liang F, Oesterreich S and Tseng GC: A joint bayesian model for integrating microarray and RNA sequencing transcriptomic data. J Comput Biol 24: 647-662, 2017.

38. Yan Y,Li S, Wang S, Rubegni P, Tognetti L, Zhang J and Yan L: Long noncoding RNA HAND2-AS1 inhibits cancer cell proliferation, migration, and invasion in esophagus squamous cell carcinoma by regulating microRNA-21. J Cell Biochem 120: 9564-9571, 2019.

39. Dong Z, Zhang A, Liu S, Lu F, Guo Y, Zhang G, Xu F, Shi Y, Shen S, Liang J and Guo W: Aberrant methylation-mediated silencing of lncRNA MEG3 functions as a ceRNA in esophageal cancer. Mol Cancer Res 15: 800-810, 2017.

40. Huang ZL, Chen RP, Zhou XT, Zhan HL, Hu MM, Liu B Wu GD and Wu LF: Long non-coding RNA MEG3 induces cell apoptosis in esophageal cancer through endoplasmic reticulum stress. Oncol Rep 37: 3093-3099, 2017.

41. Yang S, Ning Q, Zhang G, Sun H, Wang Z and Li Y: Construction of differential mRNA-lncRNA crosstalk networks based on ceRNA hypothesis uncover key roles of IncRNAs implicated in esophageal squamous cell carcinoma. Oncotarget 7: 85728-85740, 2016.

42. Zang W, Wang T, Wang Y, Chen X, Du Y, Sun Q, Li M, Dong Z and Zhao G: Knockdown of long non-coding RNA TP73-AS1 inhibits cell proliferation and induces apoptosis in esophageal squamous cell carcinoma. Oncotarget 7: 19960-19974, 2016.

43. Zhou XL, Wang WW, Zhu WG, Yu CH, Tao GZ, Wu QQ Song YQ, Pan P and Tong YS: High expression of long non-coding RNA AFAP1-AS1 predicts chemoradioresistance and poor prognosis in patients with esophageal squamous cell carcinoma treated with definitive chemoradiotherapy. Mol Carcinog 55: 2095-2105, 2016

44. Shi H, Liu Z, Pei D, Jiang Y, Zhu H and Chen B: Development and validation of nomogram based on lncRNA ZFAS1 for predicting survival in lymph node-negative esophageal squamous cell carcinoma patients. Oncotarget 8: 59048-59057, 2017.

45. Wang P, Guo Q, Gao Y, Zhi H, Zhang Y, Liu Y, Zhang J, Yue M, Guo M, Ning S, et al: Improved method for prioritization of disease associated lncRNAs based on ceRNA theory and functional genomics data. Oncotarget 8: 4642-4655, 2017.

46. Hu Q, Tai S and Wang J: Oncogenicity of lncRNA FOXD2-AS1 and its molecular mechanisms in human cancers. Pathol Res Pract 215: 843-848, 2019.

47. Stelzer Y, Sagi I, Yanuka O, Eiges R and Benvenisty N: The noncoding RNA IPW regulates the imprinted DLK1-DIO3 locus in an induced pluripotent stem cell model of Prader-Willi syndrome. Nat Genet 46: 551-557, 2014.

48. Zang Y, Zhou X, Wang Q, Li X and Huang H: LncRNA FIRRE/NF-kB feedback loop contributes to OGD/R injury of cerebral microglial cells. Biochem Biophys Res Commun 501: 131-138, 2018.

49. Su C, Shi K, Cheng X, Han Y, Li Y, Yu D and Liu Z: Long noncoding RNA LINC00472 inhibits proliferation and promotes apoptosis of lung adenocarcinoma cells via regulating miR-24-3p/ DEDD. Technol Cancer Res Treat 17: 1533033818790490, 2018.

50. Lu T, Chen D, Wang Y, Sun X, Li S, Miao S, Wo Y, Dong Y, Leng X, $\mathrm{Du} \mathrm{W}$ and Jiao W: Identification of DNA methylation-driven genes in esophageal squamous cell carcinoma: A study based on the cancer genome atlas. Cancer Cell Int 19: 52, 2019.

51. Yang W, Qu Y, Tan B, Jia Y, Wang N, Hu P and Wang J: Prognostic significance of preoperative IKBKE expression in esophageal squamous cell carcinoma. Onco Targets Ther 11: $1305-1314,2018$.

52. Maehara O, Suda G, Natsuizaka M, Ohnishi S, Komatsu Y, Sato F, Nakai M, Sho T, Morikawa K, Ogawa K, et al: Fibroblast growth factor-2-mediated FGFR/Erk signaling supports maintenance of cancer stem-like cells in esophageal squamous cell carcinoma. Carcinogenesis 38: 1073-1083, 2017. 
53. Unger JM, Vaidya R, Hershman DL, Minasian LM and Fleury ME: Systematic review and meta-analysis of the magnitude of structural, clinical, and physician and patient barriers to cancer clinical trial participation. J Natl Cancer Inst 111: 245-255, 2019.

54. Li PD, Hu JL, Ma C, Ma H, Yao J, Chen LL, Chen J, Cheng TT, Yang KY, Wu G, et al: Upregulation of the long non-coding RNA PVT1 promotes esophageal squamous cell carcinoma progression by acting as a molecular sponge of miR-203 and LASP1. Oncotarget 8: 34164-34176, 2017.

55. Bao J, Zhou C, Zhang J, Mo J, Ye Q, He J and Diao J: Upregulation of the long noncoding RNA FOXD2-AS1 predicts poor prognosis in esophageal squamous cell carcinoma. Cancer Biomark 21: $527-533,2018$
56. Kang M, Ren M,Li Y,Fu Y,Deng M and Li C: Exosome-mediated transfer of lncRNA PART1 induces gefitinib resistance in esophageal squamous cell carcinoma via functioning as a competing endogenous RNA. J Exp Clin Cancer Res 37: 171, 2018.

57. Chen LP, Wang H, Zhang Y, Chen QX, Lin TS, Liu ZQ and Zhou YY: Robust analysis of novel mRNA-lncRNA cross talk based on ceRNA hypothesis uncovers carcinogenic mechanism and promotes diagnostic accuracy in esophageal cancer. Cancer Manag Res 11: 347-358, 2018.

(i) (9) This work is licensed under a Creative Commons Attribution-NonCommercial-NoDerivatives 4.0 International (CC BY-NC-ND 4.0) License. 